\title{
A Fragment Diabatization Linear Vibronic Coupling Model for Quantum Dynamics of Multichromophoric Systems: Population of the Charge Transfer State in the Photoexcited Guanine Cytosine Pair
}

\author{
James A. Green, ${ }^{\dagger}$ Martha Yaghoubi Jouybari, ${ }^{\ddagger}$ Haritha Asha, ${ }^{\dagger}$ Fabrizio \\ Santoro, ${ }^{*, \ddagger}$ and Roberto Improta*, ${ }^{* \dagger}$ \\ $\dagger$ Consiglio Nazionale delle Ricerche, Istituto di Biostrutture e Bioimmagini (IBB-CNR), \\ via Mezzocannone 16, I-80136 Napoli, Italy \\ $\ddagger$ Consiglio Nazionale delle Ricerche, Istituto di Chimica dei Composti Organo Metallici \\ (ICCOM-CNR), SS di Pisa, Area della Ricerca, via G. Moruzzi 1, I-56124 Pisa, Italy \\ E-mail: fabrizio.santoro@pi.iccom.cnr.it; robimp@unina.it
}

\begin{abstract}
We introduce a method (FrD-LVC) based on a fragment diabatization (FrD) for the parametrization of a Linear Vibronic Coupling (LVC) model suitable for studying the photophysics of multichromophore systems. In combination with effective quantum dynamics (QD) propagations with multilayer multiconfigurational time-dependent Hartree (ML-MCTDH), the FrD-LVC approach gives access to the study of the competition between intra-chromophore decays, like those at conical intersections, and inter-chromophore processes, like exciton localization/delocalization and the involvement of charge transfer (CT) states. We used FrD-LVC parametrized with TD-DFT calculations, adopting either CAM-B3LYP or $\omega$ B97X-D functionals, to study the ultrafast photoexcited QD of a Guanine-Cytosine (GC) hydrogen bonded pair, within a Watson-Crick arrangement, considering up to 12 coupled diabatic electronic states and the effect of all the 99 vibrational coordinates. The bright excited states localized on $\mathrm{C}$ and, especially, on $\mathrm{G}$ are predicted to be strongly coupled to the $\mathrm{G} \rightarrow \mathrm{C}$ CT state which is efficiently and quickly populated after an excitation to any of the four lowest energy bright local excited states. Our QD simulations show that more than $80 \%$ of the excited population on $\mathrm{G}$ and $\sim 50 \%$ of that on $\mathrm{C}$ decays to this $\mathrm{CT}$ state in less than $50 \mathrm{fs}$. We investigate the role of vibronic effects in the population of the CT state and show it depends mainly on its large reorganization energy so that it can occur even when it is significantly less stable than the bright states in the Franck-Condon region. At the same time, we document that the formation of the GC pair almost suppresses the involvement of dark $\mathrm{n} \pi^{*}$ excited states in the photoactivated dynamics.
\end{abstract}

\section{Introduction}

The excited state dynamics of multichromophore systems rules many fundamental biochemical and technological phenomena. ${ }^{1-9}$
These systems are often described within an excitonic picture, where the focus is usually on inter-molecular processes, like the transfer of energy or charge from one chromophore to another, or the delocalization of excited states 
among many units or "sites". Model Hamiltonians have been proposed to describe these processes accounting for electronic couplings (often considered independent of the nuclear coordinates) and vibrational motions, along "tuning modes" leading toward the energy minima of the different states. ${ }^{7,8,10,11}$ However, in many cases, each single chromophore is also characterized by a rich intrinsic dynamics, with internal conversion or inter-system crossing between local excitations (LEs, such as bright $\pi \pi^{*}$ and dark $\mathrm{n} \pi^{*}$ states). For certain multichromophore systems, for example DNA, these intra-molecular decays of the individual units are actually competitive with inter-molecular processes. $^{1-4}$ When such molecular processes are ultrafast, they usually occur at Conical Intersections (CoIs) of the excited potential energy surfaces (PESs). If the chromophores are rigid enough, the essential features of the dynamics can be captured with simple vibronic coupling Hamiltonian models, ${ }^{12}$ like the linear vibronic coupling (LVC) model, where CoIs arise from the combined action of tuning modes and coupling modes.

In this contribution, we introduce an automatic parametrization of a generalized LVC model of multichromophore systems using a fragment based diabatization (FrD-LVC) that allows the investigation of the competition between intra-molecular and inter-molecular processes. LVC models have attracted renewed interest recently, as a cost effective method to study nonadiabatic spectroscopy and intramolecular excited state dynamics. ${ }^{8,9,13-28}$ On the other side, fragment-based models are popular approaches to study the excited state dynamics of multichromophore systems, ${ }^{8,9,22-31}$ but in most of their implementations they consider each single chromophore (site) as characterized by a single relevant excited state, or few states but without an internal (nonadiabatic) dynamics.

As a first test application of the FrD-LVC method, we choose to study a minimal, yet extremely relevant, system: the dimer formed by Guanine and Cytosine connected by three hydrogen bonds in a Watson and Crick (WC) arrangement, hereafter simply GC (see Fig- ure 1). GC constitutes $\sim 40 \%$ of the human genome and its photoactivated behavior has been thoroughly investigated in the gas phase, ${ }^{32-41}$ in chloroform solution, ${ }^{42-46}$ and in DNA duplexes, ${ }^{39,40,47,48}$ due to its possible involvement in the Proton Coupled Electron Transfer (PCET) processes in DNA. ${ }^{49-51}$ Timeresolved (TR) experiments in the gas phase show that when GC is in a WC arrangement, after a UV pulse, the absorption of the infrared probe is broad and featureless, suggesting an ultrashort excited state lifetime. ${ }^{52}$ According to the seminal contributions by Sobolewski, Domcke et al., a very effective PCET process is indeed operative. ${ }^{32,33}$ The lowest energy bright excited states of GC decay to an inter-molecular charge transfer (CT) quasi-dark excited state $\mathrm{G}^{+} \mathrm{C}^{-}(\mathrm{G} \rightarrow \mathrm{C})$, which, in turn, can undergo to a inter-molecular proton transfer $(\mathrm{PT})$. The latter involves the transfer of the $\mathrm{H} 1$ atom from Gua-N1 to Cyt-N3 (see Figure 1), and is the doorway for a sub-ps ground state recovery. ${ }^{32,33}$ Such combined PCET process should be operative also in low-polar solvents, ${ }^{42,43,45}$ and its involvement in DNA is also matter of debate. ${ }^{1,47,48}$ Many computational dynamics studies have thus been devoted to simulate the photoactivated dynamics of GC in different environments, ${ }^{34-36,39,40,47}$ but, to the best of our knowledge, without considering quantum nuclear effects.

In this study we focus on the first part of this process, i.e. the possible population of the $\mathrm{CT}$ state, using our FrD-LVC Hamiltonian in combination with quantum dynamics (QD) simulations to explore the excited state dynamics of GC following the excitation to the four lowest energy bright excited states. The PCET reaction occurs essentially on the PES of the CT state, ${ }^{32,33}$ therefore it is fundamental to assess on which timescale it is populated, and what are the main vibronic effects mediating this process.

This work gives us the opportunity to tackle another crucial issue in the field of the photophysics of nucleic acids, namely the effect that WC hydrogen bond pairing has on the population of the dark $n \pi^{*}$ states localized on individual nucleobases. ${ }^{1}$ Actually, there are several 
computational indications that in the gas phase a significant population transfer to the dark $\mathrm{n} \pi^{*}$ occurs for photoexcited Cytosine. ${ }^{14-17,53}$ Moreover, the spectral signature of a dark state is found for Cytosine and its derivatives in chloroform ${ }^{54}$ and in water ${ }^{55,56}$ and it has been proposed that this state is populated also within DNA duplex. ${ }^{57}$

In this contribution we thus describe our methodological approach, focusing on the new developments, and we use it to study the photoexcited state dynamics of the WC GC pair formed by 1-methylcytosine and 9-methylguanine, i.e. modeling the sugars present in the nucleotide by methyl groups. Thanks to the effectiveness of parametrization of the FrD-LVC Hamiltonian from timedependent density functional theory (TDDFT), and the potentiality of the multi-layer extension of the multiconfiguration time dependent Hartree (ML-MCTDH) method for wavepacket propagation, we included up to 12 electronic states and all the 99 vibrational modes of the system. However, since our Hamiltonian is not suited to describe CoIs at very distorted geometries, our simulations do not include the decays to the ground state, which for both the bases are known to occur at strongly non-planar CoIs on the sub-ps timescale in gas-phase. ${ }^{1}$ Therefore, we will focus on the ultrafast timescale ( $\sim 100$ fs) when large outof-plane deviations of the molecular structure are not expected, and the involvement of triplet excited states is marginal. ${ }^{58-60}$

We use TD-DFT as a reference electronic method, with two different functionals: CAMB3LYP ${ }^{61}$ and $\omega$ B97X-D,. ${ }^{62}$ These two widely adopted, long range-corrected functionals provide a different estimate of the relative stability of the lowest energy $\mathrm{G} \rightarrow \mathrm{C}$ CT state, thus providing the opportunity of checking the dependence of our predictions on this seemingly crucial parameter. Quantum dynamical simulations, starting from the four lowest energy bright excited states, predict a fast (on the $\leq 50$ fs time scale) population transfer to the lowest energy CT state, which is particularly effective ( $\geq 80 \%)$ when exciting the bright excited states of G. In contrast to what happens with isolated cytosine ${ }^{15-17}$ no significant population transfer to the dark $\mathrm{n} \pi^{*}$ states is predicted. This study provides, at full QD level, new insights on the interplay between electronic and vibrational degrees of freedom in a process of crucial biochemical interest. At the same time, it shows that FrD-LVC, thanks to its flexibility and relatively low computational cost, can be a very useful tool for the study of the photoactivated dynamics of multichromophore systems.

\section{Methods}

The approach taken in the present work is a combination of our fragment diabatization scheme to parametrize a purely electronic excitonic model in Ref. 63, and the diabatization scheme to parametrize a LVC model in Refs. 14 and 15. The LVC Hamiltonian for a coupled set of diabatic electronic states $|\boldsymbol{d}\rangle=$ $\left(\left|d_{1}\right\rangle,\left|d_{2}\right\rangle, \ldots,\left|d_{N}\right\rangle\right)$ may be written as

$$
\begin{aligned}
H= & \sum_{i}\left(K+V_{i i}^{\mathrm{d}}(\boldsymbol{q})\left|d_{i}\right\rangle\left\langle d_{i}\right|\right)+ \\
& \sum_{i, j>i} V_{i j}^{\mathrm{d}}(\boldsymbol{q})\left(\left|d_{i}\right\rangle\left\langle d_{j}|+| d_{j}\right\rangle\left\langle d_{i}\right|\right),
\end{aligned}
$$

where $\boldsymbol{q}$ are the dimensionless normal mode coordinates, defined on the ground electronic state $\mathrm{S}_{0}$, with conjugate momenta $\boldsymbol{p}$. The kinetic $K$ and potential $V$ terms of the Hamiltonian are defined as

$$
\begin{gathered}
K=\frac{1}{2} \boldsymbol{p}^{T} \boldsymbol{\Omega} \boldsymbol{p} \\
V_{i i}^{\mathrm{d}}(\boldsymbol{q})=E_{i i}^{\mathrm{d}}(0)+\boldsymbol{\lambda}_{i i}^{T} \boldsymbol{q}+\frac{1}{2} \boldsymbol{q}^{T} \boldsymbol{\Omega} \boldsymbol{q} \\
V_{i j}^{\mathrm{d}}(\boldsymbol{q})=E_{i j}^{\mathrm{d}}(0)+\boldsymbol{\lambda}_{i j}^{T} \boldsymbol{q},
\end{gathered}
$$

with $\Omega$ the diagonal matrix of normal mode frequencies $\omega_{\alpha}, \boldsymbol{\lambda}_{i j}$ the vector of linear coupling constants, $E_{i i}^{\mathrm{d}}(0)$ the diabatic energy of state $i$ at the reference geometry $(0)$, and $E_{i j}^{\mathrm{d}}(0)$ an electronic coupling constant between diabatic states $i$ and $j$ at the reference geometry. Notice that this latter term does not appear in the standard LVC approach, ${ }^{12}$ however in the FrD-LVC approach the diabatic states may be 


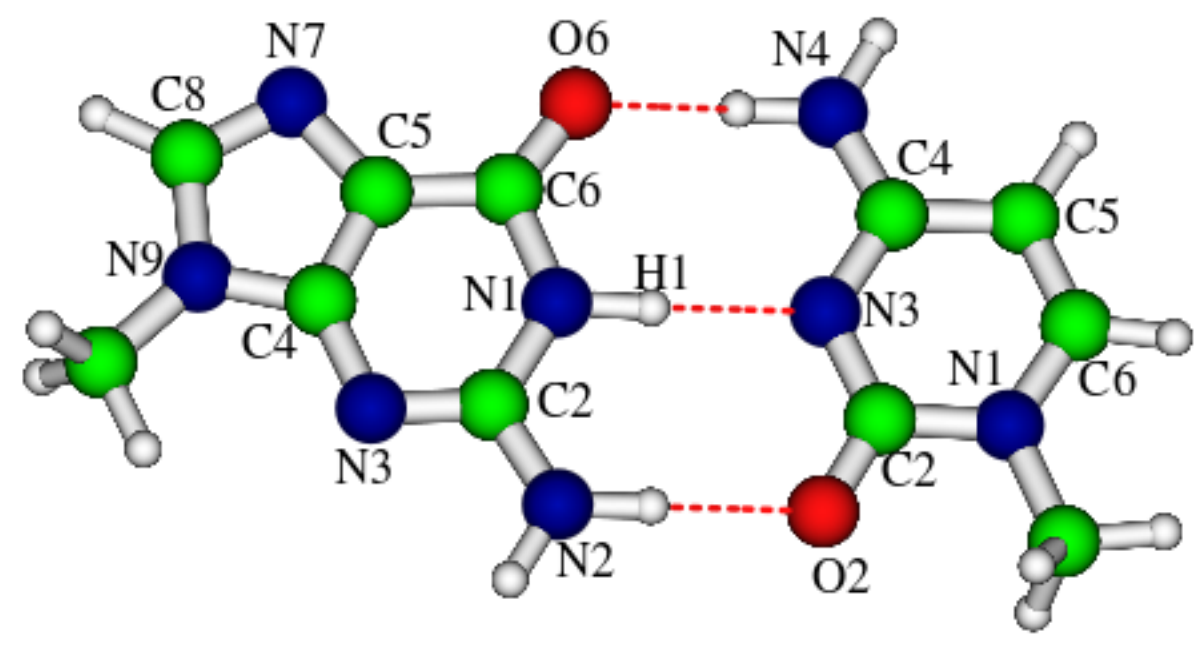

Figure 1: Schematic drawing and atom labeling of the computational model of the GuanosineCytidine dimer in a Watson-Crick arrangement. Sugar rings are modelled by the methyl groups bonded at Gua-N9 and Cyt-N1.

coupled electronically even at the reference geometry, as will be revealed below.

Following the procedure established in Ref. 63, we define diabatic states of some multichromophoric complex (MC), consisting of $N_{\text {frag }}$ fragments on the basis of reference states $\left|\boldsymbol{R}^{\text {frags }}\right\rangle$ of either the adiabatic states of the fragments (for LEs), or orbital transitions between the fragments (for CT states). The diabatic states are then obtained via a transformation of the adiabatic states of the MC

$$
\begin{aligned}
|\boldsymbol{d}\rangle & =\left|\boldsymbol{a}^{\mathrm{MC}}\right\rangle \boldsymbol{D} \\
& =\left|\boldsymbol{a}^{\mathrm{MC}}\right\rangle \boldsymbol{S}^{T}\left(\boldsymbol{S} \boldsymbol{S}^{T}\right)^{-\frac{1}{2}}
\end{aligned}
$$

where $\boldsymbol{S}=\left\langle\boldsymbol{R}^{\mathrm{frags}} \mid \boldsymbol{a}^{\mathrm{MC}}\right\rangle$ is the overlap of the reference states of the fragments with the adiabatic states of the MC. Notice that $\boldsymbol{S}$ is not necessarily a square matrix, and in general, in order to properly project the selected $N$ diabatic states one will need to consider $M$ adiabatic states at the reference geometry with $M>N$. The derivation of the transformation matrix $\boldsymbol{D}$ in terms of the overlap matrix $\boldsymbol{S}$ uses Löwdin re-orthogonalisation, and has been defined in our previous works. ${ }^{15,63}$

Performing the transformation at the reference geometry, i.e. $\boldsymbol{S}(0)=\left\langle\boldsymbol{R}^{\text {frags }}(0) \mid \boldsymbol{a}^{\mathrm{MC}}(0)\right\rangle$, yields the transformation matrix $\boldsymbol{D}(0)$. This can be applied to the diagonal matrix of adia- batic energies of the MC computed at the reference geometry

$$
\begin{gathered}
H\left[\boldsymbol{a}^{\mathrm{MC}}(0)\right]=\operatorname{diag}\left(E_{1}^{\mathrm{MC}}(0), E_{2}^{\mathrm{MC}}(0),\right. \\
\left.\ldots, E_{M}^{\mathrm{MC}}(0)\right),
\end{gathered}
$$

to obtain the diabatic matrix

$$
H[\boldsymbol{d}(0)]=\boldsymbol{D}(0)^{T} H\left[\boldsymbol{a}^{\mathrm{MC}}(0)\right] \boldsymbol{D}(0),
$$

which contains the diabatic energies $E_{i i}^{\mathrm{d}}(0)$ on the diagonal, and electronic couplings $E_{i j}^{\mathrm{d}}(0)$ on the off diagonal. In fact, while for a 'standard' LVC approach it is customary to define the $N$ diabatic states to be identical to $N$ adiabatic states at the reference geometry, in the FrDLVC approach diabatic states defined on the fragments are in general not eigenstates of the electronic Hamiltonian of the MC, and therefore they exhibit non vanishing couplings.

To obtain the linear coupling constants $\boldsymbol{\lambda}_{i j}$, we displace each normal coordinate $\alpha$ by some small value $\pm \Delta_{\alpha}$ and perform a numerical differentiation

$$
\lambda_{i j, \alpha}=\frac{\partial V_{i j}^{\mathrm{d}}(\mathbf{q})}{\partial q_{\alpha}} \simeq \frac{E_{i j}^{\mathrm{d}}\left(\Delta_{\alpha}\right)-E_{i j}^{\mathrm{d}}\left(-\Delta_{\alpha}\right)}{2 \Delta_{\alpha}} .
$$

The diabatic energies and electronic couplings at displaced geometries $E_{i j}^{\mathrm{d}}\left(\Delta_{\alpha}\right)$ are obtained by performing the diabatization based on max- 
imum overlap of the reference states with the diabatic states at displaced geometry. This amounts to computing the overlap of the reference states at equilibrium geometry with the adiabatic states of the MC at displaced geometry, i.e. $\boldsymbol{S}\left(\Delta_{\alpha}\right)=\left\langle\boldsymbol{R}^{\text {frags }}(0) \mid \boldsymbol{a}^{\mathrm{MC}}\left(\Delta_{\alpha}\right)\right\rangle$, and performing the transformation in Eq. 5. The transformation matrix at displaced geometry $\boldsymbol{D}\left(\Delta_{\alpha}\right)$, can be applied to the diagonal matrix of adiabatic energies of the MC at displaced geometry

$$
\begin{gathered}
H\left[\boldsymbol{a}^{\mathrm{MC}}\left(\Delta_{\alpha}\right)\right]=\operatorname{diag}\left(E_{1}^{\mathrm{MC}}\left(\Delta_{\alpha}\right), E_{2}^{\mathrm{MC}}\left(\Delta_{\alpha}\right),\right. \\
\left.\ldots, E_{M}^{\mathrm{MC}}\left(\Delta_{\alpha}\right)\right),
\end{gathered}
$$

to obtain the diabatic matrix at displaced geometry

$$
H\left[\boldsymbol{d}\left(\Delta_{\alpha}\right)\right]=\boldsymbol{D}\left(\Delta_{\alpha}\right)^{T} H\left[\boldsymbol{a}^{\mathrm{MC}}\left(\Delta_{\alpha}\right)\right] \boldsymbol{D}\left(\Delta_{\alpha}\right) .
$$

This diabatic Hamiltonian matrix contains $E_{i i}^{\mathrm{d}}\left(\Delta_{\alpha}\right)$ on the diagonal and $E_{i j}^{\mathrm{d}}\left(\Delta_{\alpha}\right)$ on the off diagonal, exactly analogous to Eq. 7 at the reference geometry. These values can then be used in Eq. 8 to obtain the linear coupling constants.

In principle any electronic structure method can be used for this diabatization, providing the reference states and overlap matrix can be appropriately defined. Recently, for instance, some of us adopted RASPT2/RASSCF to parametrize a LVC model to study photoexcited pyrene. ${ }^{18}$ However, as previously done for DNA nucleobases and G-quadruplexes, ${ }^{13-15,17,63}$ we define these quantities within the framework of TD-DFT. The derivation has been presented in previous papers, ${ }^{15,63}$ and we also include it in the Supporting Information (SI, Section S1.1), as we have found a more computationally efficient method of calculating the overlap matrix.

For the definition of the reference geometry and normal mode coordinates, in principle two choices could be made: (i) define the reference geometry as the ground state equilibrium geometry of the $\mathrm{MC}$, and then the normal coordinates as those of the entire $\mathrm{MC}$, or (ii) define the reference geometry as any arrangement of individual fragments, not necessarily at the equilibrium geometry of the overall $\mathrm{MC}$, but with the individual fragments at their own equilibrium geometry. Then, the normal mode coordinates are those of the individual fragments. Option (i) is appropriate when vibrational modes are strongly coupled between fragments, such as when strong hydrogen bonds are present. The disadvantage would be an expensive optimisation and frequency calculation for a large MC. Option (ii) is appropriate when the converse is true, and vibrational modes are generally localized to individual sites. This therefore neglects low frequency inter-fragment vibrations, however they could be re-introduced by defining a proper number of degrees of freedom describing such vibrations between rigid monomers. It should be noted that option (ii) would still capture the vibronic coupling between excitations located on different sites that are due to the vibrations of a single site. One could also imagine a combination of options (i) and (ii), where the entire MC is divided into sub-systems of multiple fragments, and the sub-system is parametrized according to option (i) and then the overall MC according to option (ii). Indeed, the flexibility of the fragment diabatization approach allows one to choose the number of fragments (and number of states) to include to best balance accuracy and efficiency, as was demonstrated in our excitonic model application. ${ }^{63}$

In the present application of the GC pair, we choose option (i) due to the relatively small size of the system, and the strong hydrogen bonds between the pair leading to vibrational modes that are not localized on either site. ${ }^{64}$ The reference geometry is therefore the ground state equilibrium geometry of GC. The FrDLVC approach is schematically shown in Figure. 2, summarizing the main steps of our procedure, i.e. (1) the choice of reference states, (2) projection of the reference states onto the $\mathrm{MC}$ at reference geometry, and (3) projection onto the $\mathrm{MC}$ at displaced geometries.

Our approach bears some similarity to the work of Tamura and Burghardt on conjugated polymers and fullerene systems, ${ }^{8,9,22-28}$ however there are a number of differences in the implementation. Firstly, we calculate the overlap $\boldsymbol{S}$ via transition densities following Neugebauer, ${ }^{65}$ rather than Slater determinants. Secondly, we 


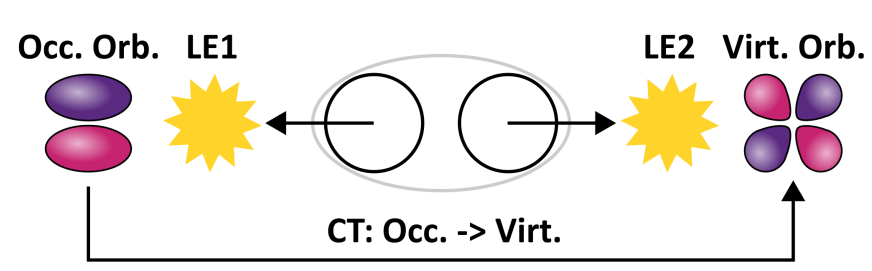

1. Calculate LEs of individual
chromophores and define CT
states as one electron
transitions from occupied to
virtual orbitals on individual
chromophores

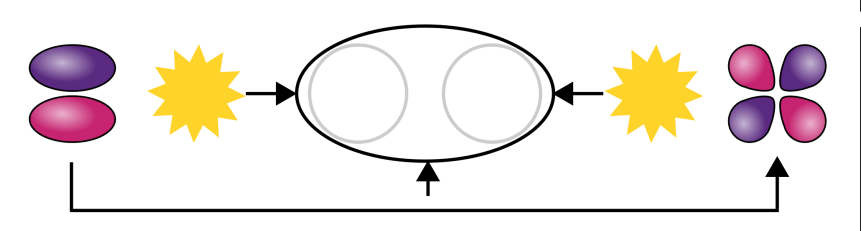

2. Project these LE and CT reference states onto adiabatic states of MC at reference geometry to obtain $E_{i i}^{\mathrm{d}}(0)$ and $E_{i j}^{\mathrm{d}}(0)$

(i)

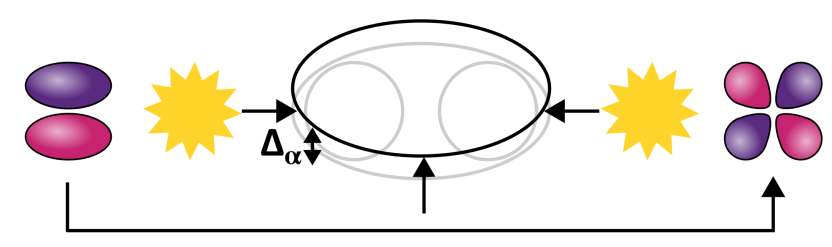

(ii)

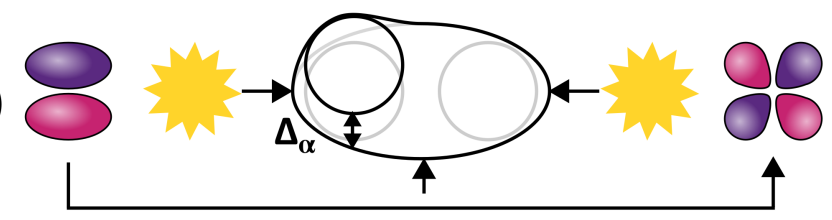

3. Displace either: (i) the normal modes of the MC or (ii) the normal modes of the individual chromophores by $\Delta_{\alpha}$, project the reference LE and $\mathrm{CT}$ states onto this structure of the MC and perform a numerical differentiation to obtain $\lambda_{i i}$ and $\lambda_{i j}$

Figure 2: Schematic of the FrD-LVC approach, illustrated with two chromophores (circles) and the $\mathrm{MC}$ consisting of these two chromophores (oval). Yellow stars represent reference LEs of individual chromophores, whilst a CT reference state is represented by an arrow from an occupied orbital of one individual chromophore to a virtual orbital of the other (1). Projections of these reference states onto the MC are shown by arrows towards the MC at either the reference (2) or displaced (3) geometries. The moiety (i.e single chromophore or MC) involved in a given computational step (excited state calculation, projection, or normal mode displacement) is depicted in black, the one not involved in gray. Illustration of the diabatic Hamiltonian shown in the centre.

do not use the "electron-hole" formalism to describe local excitations. In this way we account for the possible existence of multi-orbital components in the local excitations a case which, for instance, does occur in DNA nucleobases. Thirdly, we include a vibrational dependence on the diabatic couplings through the $\boldsymbol{\lambda}_{i j}$ parameter, and fourthly, we compute the reference states based on isolated chromophores, rather than well-separated ones.

\section{Computational Details}

Electronic structure calculations have been performed with DFT for the ground state, and TD-DFT for the excited states, using two different range-separated functionals that confer different CT state stability, CAM-B3LYP ${ }^{61}$ and $\omega \mathrm{B} 97 \mathrm{X}-\mathrm{D},{ }^{62}$ and two basis sets 6-31G(d) and 6$31+\mathrm{G}(\mathrm{d}, \mathrm{p})$, using the Gaussian 16 program. ${ }^{66}$ The 6-31G(d) basis set is used in parametrization of the FrD-LVC models presented in the main text. Single point energy test calcula- 
tions are performed with the $6-31+\mathrm{G}(\mathrm{d}, \mathrm{p})$ basis set for both functionals, which is also used to build a test FrD-LVC model with the $\omega$ B97X$\mathrm{D}$ functional. These results are shown in the SI, Section S6, to check the solidity of our conclusion with respect to an increase of the size of the basis set. TD-DFT computations were performed using tight SCF convergence, with a $10^{-6}$ a.u. threshold.

As a molecular model, we use 9-methylguanine and 1-methylcytosine to represent GC in Watson Crick conformation (see Figure 1), geometry optimised with $C_{\mathrm{s}}$ symmetry, which permitted electronic decoupling of the $\mathrm{A}^{\prime}\left(\pi \pi^{*}\right.$ and CT) and $\mathrm{A}^{\prime \prime}\left(\mathrm{n} \pi^{*}\right)$ states. The vibrational frequencies obtained in the $\mathrm{S}_{0}$ state of GC are utilised for each of the excited states in the FrD-LVC models. Two different FrDLVC models are parametrized for each of the functionals: (i) a 12 diabatic state model, including $4 \pi \pi^{*}, 2 \mathrm{CT}$ and $6 \mathrm{n} \pi^{*}$ states and both in-plane (A') and out of plane (A") modes for a full-dimensional (99 mode) picture of GC, and (ii) a reduced-dimensionality (65 mode) 5 state model, including $4 \pi \pi^{*}$ and 1 CT state, so that out-of-plane A" modes are not active and have been neglected. These diabatic states were defined based on reference states of individual $\mathrm{G}$ and $\mathrm{C}$ at the same geometry as in the GC pair, using the procedure described in Section 2, and then projected onto the calculation of 40 adiabatic states of GC by TD-DFT (see Eq. 5). The diabatization was performed with an in-house code interfaced with Gaussian 16 that is freely available upon request. LVC models for individual $\mathrm{G}$ and $\mathrm{C}$ have also been parametrized at the same level of theory as GC, including $2 \pi \pi^{*}$ and $3 \mathrm{n} \pi^{*}$ states for each base, following the procedure for individual bases we have recently used. ${ }^{15-17}$

Quantum dynamics calculations were performed with the ML-MCTDH method, ${ }^{67-69}$ adopting the implementation within the Quantics package. ${ }^{70}$ We used a variable mean field (VMF) with a Runge-Kutta integrator of order 5 and accuracy $10^{-7}$, as in the provided examples for $S_{2} / S_{1}$ dynamics of pyrazine with 24 normal modes. ${ }^{68,71}$ For the primitive basis set we adopted Hermite DVR functions, as ap- propriate for harmonic potentials. We checked convergence by monitoring the populations at the beginning and end of the grid using the rdgpop tool provided in Quantics, and ensuring that they did not exceed $10^{-9}$. For the ML "tree" expansion, we chose the number of single particle functions (SPFs) for each node based on the magnitude of the linear coupling constants $\lambda_{i i, \alpha}$, with modes with larger couplings assigned larger numbers of SPFs, as we have done in recent studies of single nucleobases. ${ }^{13-17}$ Mode combination was also utilised for modes with similar character. The eigenvalues of the density matrices of each node in the ML tree, also known as the natural weights, were monitored, ensuring that the smallest natural weight was always less than $1 \%$ to obtain a reasonable quality calculation, as indicated in the Quantics manual.

Absorption spectra of GC, G, and C were calculated following the procedure we have recently utilised, via the Fourier transform of the auto-correlation function produced by the quantum dynamics calculations, weighted by the transition dipoles. ${ }^{17,19}$ For GC, the transition dipoles of the diabatic states were obtained by application of the transformation matrix $\boldsymbol{D}(0)$ to the adiabatic transition dipole moments obtained by TD-DFT at the FranckCondon (FC) point, in the same manner as Eq. 7 and as previously described in Ref. 63. For monomeric $\mathrm{G}$ and $\mathrm{C}$, the diabatic transition dipoles are equivalent to the adiabatic TD-DFT ones. The absorption spectra include contributions from initial excitation to each of the $\pi \pi^{*}$ states, and are phenomenologically broadened with a Gaussian of half-width half-maximum $\mathrm{HWHM}=0.04 \mathrm{eV}$. Further details may be found in the SI, Section S1.2.

Expectation values of the FrD-LVC diabatic potential diagonal $\left(V_{i i}^{\mathrm{d}}\right)$ and off diagonal $\left(V_{i j}^{\mathrm{d}}\right)$ terms are also calculated by integrating the wavepacket over all normal modes. Since each diabatic potential is the sum of independent terms on the different modes $V_{i j}^{\mathrm{d}}(\mathbf{q})=$ $\sum_{\alpha} V_{i j}^{\mathrm{d}}\left(q_{\alpha}\right)$, it is straightforward to define the expectation value of such potential terms $\left\langle\Psi\left|V_{i j}^{\mathrm{d}}\left(q_{\alpha}\right)\right| \Psi\right\rangle$ on each mode. 


\section{Results}

\subsection{Adiabatic and diabatic states: FC point and minima}

The 12 lowest singlet state energies of GC, calculated by the CAM-B3LYP and $\omega$ B97X-D functionals and 6-31G(d) basis set are shown in Table 1 (TD-DFT column). WC pairing leads to a non-negligible mixing between the $\pi \pi^{*}$ states on $\mathrm{G}$ and $\mathrm{C}$ (especially according to $\omega \mathrm{B} 97 \mathrm{X}-\mathrm{D})$, and the $\mathrm{CT}$ state, as shown with the natural transition orbitals (NTOs) in the SI, Figures S1 and S2. Nonetheless, we can identify $2 \pi \pi^{*}$ states on C, 2 $\pi \pi^{*}$ states on $\mathrm{G}$ (commonly referred to as $\mathrm{L}_{\mathrm{a}}$ and $\mathrm{L}_{\mathrm{b}}$ following Platt's nomenclature), $3 \mathrm{n} \pi^{*}$ states of $\mathrm{C}, 3 \mathrm{n} \pi^{*}$ states of $\mathrm{G}$, and $2 \mathrm{G} \rightarrow \mathrm{C}$ $\mathrm{CT}$ states. The $\mathrm{G} \rightarrow \mathrm{C}(\mathrm{CT}) 1$ state involves a $\operatorname{HOMO}(\mathrm{G}) \rightarrow \mathrm{LUMO}(\mathrm{C})$ transition, whilst the $\mathrm{G} \rightarrow \mathrm{C}(\mathrm{CT}) 2$ state a $\mathrm{HOMO}(\mathrm{G}) \rightarrow \mathrm{LUMO}+1(\mathrm{C})$ transition. Considering the differences in the basis set, CAM-B3LYP and, especially, wB97X$\mathrm{D}$ provide a description of the $\mathrm{FC}$ region in good agreement with the EOM-CCSD(T) results of Ref. 72 that are also shown in the Table.

The ordering and relative energy gaps between the states is quite similar for $\omega \mathrm{B} 97 \mathrm{X}-$ $\mathrm{D}$ and $\operatorname{EOM-CCSD}(\mathrm{T})$, with the main difference being that the stability of the $\mathrm{C}\left(\pi \pi^{*} 2\right)$ and $\mathrm{G}\left(\mathrm{L}_{\mathrm{b}}\right)$ states is switched. For CAM-B3LYP, as well as the $\mathrm{C}\left(\pi \pi^{*} 2\right)$ and $\mathrm{G}\left(\mathrm{L}_{\mathrm{b}}\right)$ switch, the $\mathrm{G} \rightarrow \mathrm{C}(\mathrm{CT}) 1$ state is predicted to be the lowest energy excited state, and its relative stability to the other bright states is overestimated by $\sim 0.45 \mathrm{eV}$ and $\sim 0.65 \mathrm{eV}$ compared to $\omega \mathrm{B} 97 \mathrm{X}-\mathrm{D}$ and EOM-CCSD $(\mathrm{T})$, respectively. The comparison with the results obtained for the isolated bases (see Ref. 17 and SI Table S1) shows that WC pairing strongly destabilizes the $\mathrm{n} \pi^{*}$ states, and, in particular the $\mathrm{G}\left(\mathrm{n}_{\mathrm{O}} \pi^{*}\right)$ and $\mathrm{C}\left(\mathrm{n}_{\mathrm{O}} \pi^{*}\right)$ states. Confirming a trend already discussed in the literature, ${ }^{1,73}$ the transfer of an electron from the lone pair involved in a hydrogen bond towards a $\pi^{*}$ delocalized on the ring weakens the hydrogen bonds and destabilizes the associated excited states. The strong hydrogen bonding is also responsible for the mix-

Table 1: Energies $(\mathrm{eV})$ of the diabatic states from the FrD-LVC 12 state models $E_{i i}^{\mathrm{d}}(0)$, compared with adiabatic energies with same predominant character from TD-DFT and via diagonalization of the FrD-LVC Hamiltonian (see text for details). State ordering in parentheses. Calculated on GC in $C_{\mathrm{s}}$ symmetry at equilibrium geometry by CAM-B3LYP/6-31G(d) and $\omega$ B97X-D/6-31G(d). Also shown are EOM-CCSD(T)/TZVP results from Ref. 72.

\begin{tabular}{l|ccc|ccc|c} 
& \multicolumn{3}{|c|}{ CAM-B3LYP } & \multicolumn{3}{|c}{$\omega$ B97X-D } & \\
\hline Diab. State & $E_{i i}^{\mathrm{d}}(0)$ & Ad. LVC & TD-DFT & $E_{i i}^{\mathrm{d}}(0)$ & Ad. LVC & TD-DFT & EOM-CCSD $(\mathrm{T})$ \\
\hline $\mathrm{G} \rightarrow \mathrm{C}(\mathrm{CT}) 1$ & 5.17 & $5.12\left(\mathrm{~S}_{1}\right)$ & $5.07\left(\mathrm{~S}_{1}\right)$ & 5.54 & $5.56\left(\mathrm{~S}_{3}\right)$ & $5.50\left(\mathrm{~S}_{3}\right)$ & 5.36 \\
$\mathrm{C}\left(\pi \pi^{*} 1\right)$ & 5.33 & $5.29\left(\mathrm{~S}_{2}\right)$ & $5.27\left(\mathrm{~S}_{2}\right)$ & 5.31 & $5.25\left(\mathrm{~S}_{1}\right)$ & $5.28\left(\mathrm{~S}_{2}\right)$ & 4.92 \\
$\mathrm{G}\left(\mathrm{L}_{\mathrm{a}}\right)$ & 5.42 & $5.36\left(\mathrm{~S}_{3}\right)$ & $5.31\left(\mathrm{~S}_{3}\right)$ & 5.42 & $5.31\left(\mathrm{~S}_{2}\right)$ & $5.21\left(\mathrm{~S}_{1}\right)$ & 4.85 \\
$\mathrm{G}\left(\mathrm{L}_{\mathrm{b}}\right)$ & 5.66 & $5.75\left(\mathrm{~S}_{4}\right)$ & $5.69\left(\mathrm{~S}_{4}\right)$ & 5.65 & $5.74\left(\mathrm{~S}_{4}\right)$ & $5.69\left(\mathrm{~S}_{4}\right)$ & 5.48 \\
$\mathrm{C}\left(\pi \pi^{*} 2\right)$ & 5.92 & $5.96\left(\mathrm{~S}_{7}\right)$ & $5.93\left(\mathrm{~S}_{7}\right)$ & 5.89 & $5.93\left(\mathrm{~S}_{6}\right)$ & $5.90\left(\mathrm{~S}_{6}\right)$ & 5.37 \\
$\mathrm{G} \rightarrow \mathrm{C}(\mathrm{CT}) 2$ & 6.39 & $6.40\left(\mathrm{~S}_{8}\right)$ & $6.33\left(\mathrm{~S}_{8}\right)$ & 6.67 & $6.68\left(\mathrm{~S}_{11}\right)$ & $6.60\left(\mathrm{~S}_{11}\right)$ & \\
$\mathrm{C}\left(\mathrm{n}_{\mathrm{N}} \pi^{*}\right)$ & 5.92 & $5.92\left(\mathrm{~S}_{5}\right)$ & $5.79\left(\mathrm{~S}_{5}\right)$ & 5.88 & $5.88\left(\mathrm{~S}_{5}\right)$ & $5.77\left(\mathrm{~S}_{5}\right)$ & 5.65 \\
$\mathrm{G}\left(\mathrm{n}_{\mathrm{O}} \pi^{*}\right)$ & 5.94 & $5.93\left(\mathrm{~S}_{6}\right)$ & $5.93\left(\mathrm{~S}_{6}\right)$ & 5.95 & $5.94\left(\mathrm{~S}_{7}\right)$ & $5.93\left(\mathrm{~S}_{7}\right)$ & 5.76 \\
$\mathrm{G}\left(\mathrm{n}_{\mathrm{N}} \pi^{*}\right)$ & 6.50 & $6.46\left(\mathrm{~S}_{10}\right)$ & $6.44\left(\mathrm{~S}_{9}\right)$ & 6.47 & $6.43\left(\mathrm{~S}_{8}\right)$ & $6.43\left(\mathrm{~S}_{8}\right)$ & \\
$\mathrm{C}\left(\mathrm{n}_{\mathrm{O}} \pi^{*}\right)$ & 6.54 & $6.46\left(\mathrm{~S}_{9}\right)$ & $6.45\left(\mathrm{~S}_{10}\right)$ & 6.53 & $6.47\left(\mathrm{~S}_{9}\right)$ & $6.46\left(\mathrm{~S}_{9}\right)$ & 6.27 \\
$\mathrm{C}\left(\mathrm{n}_{\mathrm{O}} \pi^{*}\right)$ & 6.55 & $6.64\left(\mathrm{~S}_{11}\right)$ & $6.60\left(\mathrm{~S}_{11}\right)$ & 6.53 & $6.59\left(\mathrm{~S}_{10}\right)$ & $6.56\left(\mathrm{~S}_{10}\right)$ & 6.42 \\
$\mathrm{G}\left(\mathrm{n}_{\mathrm{N}} \pi^{*} 2\right)$ & 6.73 & $6.78\left(\mathrm{~S}_{12}\right)$ & $6.71\left(\mathrm{~S}_{12}\right)$ & 6.73 & $6.77\left(\mathrm{~S}_{12}\right)$ & $6.70\left(\mathrm{~S}_{12}\right)$ &
\end{tabular}


ing between the different excited states, as well as the close proximity of the bases.

Single point energy calculations with the larger $6-31+G(d, p)$ basis set are shown in the SI, Table S14. They reveal a very similar energy ordering, and the changes in the relative stability of the different states are small. See Section S6 of the SI for further details on the $6-31+\mathrm{G}(\mathrm{d}, \mathrm{p})$ basis set test.

Also shown in Table 1 are the energies of the diabatic states at the $\mathrm{FC}$ point, $E_{i i}^{\mathrm{d}}(0)$, calculated by the FrD procedure and used in the LVC model. In contrast to the TD-DFT states, by construction our LEs are fully localized either on G or on C. The mixing of LE and CT states at the $\mathrm{FC}$ point is accounted for through the constant coupling parameter $E_{i j}^{\mathrm{d}}(0)$, see Table 2 for a selected few, and Tables S2 and S3 in the SI for all. The energies of the adiabatic states of the FrD-LVC model at the FC point, i.e. the eigenvalues of the FrD-LVC Hamiltonian, are reported in the 'Ad. LVC' column of Table 1 (the corresponding eigenvectors are shown in the SI, Tables S8 and S9). These energies are within $\sim 0.05-0.1 \mathrm{eV}$ of the TD-DFT energies, and in predominantly the same order, indicat- ing that our model appropriately reproduces the low-energy adiabatic states of the dimer at the FC point. The only exceptions are the ordering of the $\mathrm{G}\left(\mathrm{n}_{\mathrm{N}} \pi^{*} 1\right)$ and $\mathrm{C}\left(\mathrm{n}_{\mathrm{O}} \pi^{*} 1\right)$ states in the CAM-B3LYP model, and the $\mathrm{C}\left(\pi \pi^{*} 1\right)$ and $\mathrm{G}\left(\mathrm{L}_{\mathrm{a}}\right)$ states in the $\omega \mathrm{B} 97 \mathrm{X}-\mathrm{D}$ model. The energies, however, are not greatly different, and it should be reminded that the TD-DFT C $\left(\pi \pi^{*} 1\right)$ and $\mathrm{G}\left(\mathrm{L}_{\mathrm{a}}\right) \omega \mathrm{B} 97 \mathrm{X}-\mathrm{D}$ states are significantly mixed.

Inspection of Table 2 and S2 in the SI shows that diabatic states localized on $\mathrm{G}$ are more significantly coupled with $\mathrm{G} \rightarrow \mathrm{C} \mathrm{CT}$ states. In particular, $\mathrm{G}\left(\mathrm{L}_{\mathrm{a}}\right)$ is the excited state with the largest coupling with $\mathrm{G} \rightarrow \mathrm{C}(\mathrm{CT}) 1$, almost twice as large as those involving the other bright excited states. A similar trend is found for $\mathrm{G} \rightarrow \mathrm{C}(\mathrm{CT}) 2$, though in this case, the most coupled state is $\mathrm{G}\left(\mathrm{L}_{\mathrm{b}}\right)$. Interestingly, $\omega \mathrm{B} 97 \mathrm{X}$ $\mathrm{D}$ and CAM-B3LYP provides a similar picture, i.e. $\mathrm{G}\left(\mathrm{L}_{\mathrm{a}}\right)$ has the largest coupling with $\mathrm{G} \rightarrow \mathrm{C}(\mathrm{CT}) 1$, notwithstanding that according to $\omega \mathrm{B} 97 \mathrm{X}-\mathrm{D}$ the $\mathrm{G} \rightarrow \mathrm{C}(\mathrm{CT}) 1$ state is energetically closer to other bright excited states. Our analysis thus suggests that 'hole' coupling is most important than the 'electron' one. In other words,

Table 2: $\pi \pi^{*}-\mathrm{CT}$, excitonic, and intra-monomer $\pi \pi^{*}$ mixing couplings between diabatic states at the FC point $\left(E_{i j}^{\mathrm{d}}(0), \mathrm{eV}\right)$ as predicted by the 12 state FrD-LVC models parametrized by CAM-B3LYP and $\omega \mathrm{B} 97 \mathrm{X}-\mathrm{D}$.

\begin{tabular}{lrr} 
Coupling & CAM-B3LYP & $\omega$ B97X-D \\
\hline & \multicolumn{2}{r}{$r \pi^{*}-\mathrm{CT}$} \\
$\mathrm{G}\left(\mathrm{L}_{\mathrm{a}}\right): \mathrm{G} \rightarrow \mathrm{C}(\mathrm{CT}) 1$ & 0.065 & 0.067 \\
$\mathrm{G}\left(\mathrm{L}_{\mathrm{b}}\right): \mathrm{G} \rightarrow \mathrm{C}(\mathrm{CT}) 1$ & 0.038 & 0.037 \\
$\mathrm{C}\left(\pi \pi^{*} 1\right): \mathrm{G} \rightarrow \mathrm{C}(\mathrm{CT}) 1$ & 0.022 & 0.023 \\
$\mathrm{C}\left(\pi \pi^{*} 2\right): \mathrm{G} \rightarrow \mathrm{C}(\mathrm{CT}) 1$ & -0.039 & -0.036 \\
& $\underline{\text { Excitonic }}$ & \\
$\mathrm{G}\left(\mathrm{L}_{\mathrm{a}}\right): \mathrm{C}\left(\pi \pi^{*} 1\right)$ & 0.002 & 0.002 \\
$\mathrm{G}\left(\mathrm{L}_{\mathrm{a}}\right): \mathrm{C}\left(\pi \pi^{*} 2\right)$ & 0.053 & 0.052 \\
$\mathrm{G}\left(\mathrm{L}_{\mathrm{b}}\right): \mathrm{C}\left(\pi \pi^{*} 1\right)$ & -0.020 & -0.018 \\
$\mathrm{G}\left(\mathrm{L}_{\mathrm{b}}\right): \mathrm{C}\left(\pi \pi^{*} 2\right)$ & 0.041 & 0.037 \\
& $\frac{\pi \pi^{*} \text { Mixing }}{2}$ & \\
$\mathrm{G}\left(\mathrm{L}_{\mathrm{a}}\right): \mathrm{G}\left(\mathrm{L}_{\mathrm{b}}\right)$ & -0.171 & -0.176 \\
$\mathrm{C}\left(\pi \pi^{*} 1\right): \mathrm{C}\left(\pi \pi^{*} 2\right)$ & 0.160 & 0.155
\end{tabular}


Table 3: Energies (eV, relative to $\mathrm{S}_{0}$ minimum) of adiabatic (coordinates $\boldsymbol{q}_{\mathrm{min}}^{\mathrm{ad}, m}$ ) and diabatic (coordinates $\boldsymbol{q}_{\min }^{\mathrm{d}, i}$ ) excited state planar minima. In each minima, the diabatic energies from the FrD-LVC model are shown $\left(V_{i i}^{\mathrm{d}}(\boldsymbol{q})\right.$ column) as well as the adiabatic energies predicted by TD-DFT, and the adiabatic energies from the FrD-LVC model (Ad. LVC column). Also shown is the RMSD between the Cartesian coordinates of the $\boldsymbol{q}_{\min }^{\mathrm{ad}, m}$ and $\boldsymbol{q}_{\min }^{\mathrm{d}, i}$ geometries in $\AA$. Further details in text.

\begin{tabular}{|c|c|c|c|c|c|c|c|}
\hline \multirow[b]{2}{*}{ State } & \multicolumn{3}{|c|}{$\boldsymbol{q}_{\mathrm{min}}^{\mathrm{ad}, m}$} & \multicolumn{3}{|c|}{$\boldsymbol{q}_{\mathrm{min}}^{\mathrm{d}, i}$} & \multirow[b]{2}{*}{ RMSD } \\
\hline & $V_{i i}^{\mathrm{d}}(\boldsymbol{q})$ & Ad. LVC & TD-DFT & $V_{i i}^{\mathrm{d}}(\boldsymbol{q})$ & Ad. LVC & TD-DFT & \\
\hline \multicolumn{8}{|c|}{ CAM-B3LYP } \\
\hline $\mathrm{G} \rightarrow \mathrm{C}(\mathrm{CT}) 1$ & 4.04 & 4.03 & 4.00 & 3.94 & 3.93 & 4.00 & 0.060 \\
\hline $\mathrm{C}\left(\pi \pi^{*} 1\right)$ & 4.95 & 4.95 & 4.94 & 4.94 & 4.95 & 4.95 & 0.007 \\
\hline $\mathrm{G}\left(\mathrm{L}_{\mathrm{a}}\right)$ & 5.18 & 5.11 & 5.07 & 5.09 & 5.03 & 5.01 & 0.052 \\
\hline $\mathrm{G}\left(\mathrm{n}_{\mathrm{O}} \pi^{*}\right)$ & 5.57 & 5.56 & 5.42 & 5.50 & 5.50 & 5.45 & 0.054 \\
\hline $\mathrm{G}\left(\mathrm{n}_{\mathrm{N}} \pi^{*} 1\right)$ & 6.02 & 5.79 & 5.68 & 5.93 & 5.81 & 5.74 & 0.030 \\
\hline $\mathrm{C}\left(\mathrm{n}_{\mathrm{O}} \pi^{*} 1\right)$ & 5.91 & 5.56 & 5.40 & 5.66 & 5.57 & 5.54 & 0.052 \\
\hline \multicolumn{8}{|c|}{$\underline{\omega \mathrm{B} 97 \mathrm{X}-\mathrm{D}}$} \\
\hline $\mathrm{G} \rightarrow \mathrm{C}(\mathrm{CT}) 1$ & 4.48 & 4.47 & 4.40 & 4.39 & 4.39 & 4.36 & 0.045 \\
\hline $\mathrm{C}\left(\pi \pi^{*} 1\right)$ & 4.94 & 4.94 & 4.91 & 4.93 & 4.92 & 4.92 & 0.006 \\
\hline $\mathrm{G}\left(\mathrm{L}_{\mathrm{a}}\right)$ & 5.21 & 5.06 & 5.02 & 5.10 & 5.07 & 5.05 & 0.039 \\
\hline $\mathrm{G}\left(\mathrm{n}_{\mathrm{O}} \pi^{*}\right)$ & 5.59 & 5.59 & 5.44 & 5.53 & 5.52 & 5.47 & 0.059 \\
\hline $\mathrm{G}\left(\mathrm{n}_{\mathrm{N}} \pi^{*} 1\right)$ & 5.94 & 5.73 & 5.65 & 5.87 & 5.75 & 5.69 & 0.025 \\
\hline $\mathrm{C}\left(\mathrm{n}_{\mathrm{O}} \pi^{*} 1\right)$ & 5.70 & 5.48 & 5.36 & 5.56 & 5.48 & 5.45 & 0.044 \\
\hline
\end{tabular}

that the LUMO's of $\mathrm{G}$ and $\mathrm{C}$ are more coupled than the HOMO's.

Table 2 also shows that hydrogen bonding leads to a significant intra-monomer coupling between the bright excited states localized on $\mathrm{G}$ and C, i.e. $\mathrm{G}\left(\mathrm{L}_{\mathrm{a}}\right)-\mathrm{G}\left(\mathrm{L}_{\mathrm{b}}\right)$ and $\mathrm{C}\left(\pi \pi^{*} 1\right)-\mathrm{C}\left(\pi \pi^{*} 2\right)$, which we refer to as $\pi \pi^{*}$ mixing couplings. This effect, which we have discussed in detail in Ref. 63 , mirrors the changes in the shape of the excited states of the isolated chromophore due to surrounding molecules and is crucial for the application of excitonic Hamiltonians to closely spaced MC assemblies. In contrast, the intermonomer excitonic couplings, i.e. those possibly promoting energy transfer between the $\pi \pi^{*}$ states are much smaller, in particular for $G\left(L_{a}\right)$ $\mathrm{C}\left(\pi \pi^{*} 1\right)$.

For the $n \pi^{*}$ states, as shown in Table S3 in the SI, hydrogen bonding affects the intra-monomer mixing couplings in a similar manner to the $\pi \pi^{*}$ states, in particular for $\mathrm{G}\left(\mathrm{n}_{\mathrm{N}} \pi^{*} 1\right)-\mathrm{G}\left(\mathrm{n}_{\mathrm{N}} \pi^{*} 2\right)$ and $\mathrm{C}\left(\mathrm{n}_{\mathrm{O}} \pi^{*} 1\right)-\mathrm{C}\left(\mathrm{n}_{\mathrm{O}} \pi^{*} 2\right)$ mixing. Whilst the inter-monomer couplings of the $\mathrm{n} \pi^{*}$ states are relatively small, consulting the eigenvectors of the $\mathrm{n} \pi^{*}$ FrD-LVC adiabatic states in Table S9 shows some mixing of the states localized on $\mathrm{G}$ and $\mathrm{C}$ at the $\mathrm{FC}$ point, in particular for the CAM-B3LYP model, due to the closeness in energy of the $\mathrm{n} \pi^{*}$ diabatic states. This indicates that the hydrogen bonding could induce a small amount of delocalization of the dark states.

The general trends in terms of the strength of coupling are confirmed when considering the effect of vibrations, which allows the coupling between $\pi \pi^{*}$ and $\mathrm{n} \pi^{*}$ states. Interestingly, as shown in Tables S12 and S13 in the SI, $\mathrm{n} \pi^{*}$ states localized on $\mathrm{C}$ are significantly vibronically coupled with $\mathrm{G} \rightarrow \mathrm{C}(\mathrm{CT}) 1$.

As an additional characterisation and test of the robustness of our approach, we have compared the energies and geometries of planar adiabatic excited state minima from TD-DFT geometry optimisations $\left(\boldsymbol{q}_{\min }^{\text {ad, } m}\right.$ for adiabatic state $m$, see also Section S2.2 and S3.2.2 in the SI) 
with the diabatic minima predicted by the FrDLVC models $\left(\boldsymbol{q}_{\min }^{\mathrm{d}, i}\right.$ for diabatic state $\left.i\right)$. As shown in Table 3, the geometries (see the low root-mean-square-deviation, RMSD) and relative energy of the two different sets of minima are quite similar.

Moreover, in general, in its minimum each adiabatic state has a more predominant contribution from a single diabatic state than in the FC region, as shown by the comparison of the eigenvectors of the diabatization procedure in the different structures (see Tables S8-S11 in the SI). For example, at the FC point the $\mathrm{S}_{1}$ and $\mathrm{S}_{2}$ states of $\omega$ B97X-D have significant mixing of the $\pi \pi^{*}$ states localized on $\mathrm{G}$ with those localized on $\mathrm{C}$ (Table S8). However, at both $\boldsymbol{q}_{\text {min }}^{\text {ad, } m}$ and $\boldsymbol{q}_{\text {min }}^{\mathrm{d}, i}$ geometries of the $\mathrm{G}\left(\mathrm{L}_{\mathrm{a}}\right)$ and $\mathrm{C}\left(\pi \pi^{*} 1\right)$ states, the FrD-LVC adiabatic states are instead predominantly composed of either $\mathrm{G}\left(\mathrm{L}_{\mathrm{a}}\right)$ or $\mathrm{C}\left(\pi \pi^{*} 1\right)$ diabatic states (Tables S10 and S11, respectively). These results support the physical significance of the 'monomer-like' diabatic states, as well as benefitting the interpretation of the dynamics results in the following section.

\subsection{Excited state dynamics of GC}

In Figure 3 we report the excited state dynamics computed by using a FrD-LVC Hamiltonian including 12 states (solid lines) and parametrized with CAM-B3LYP or $\omega$ B97X-D for initial excitations to each of the four lowest bright excited states. When exciting $G\left(L_{a}\right)$, CAM-B3LYP predicts that, after an ultrashort 'spike' due to the coupling with $\mathrm{G}\left(\mathrm{L}_{\mathrm{b}}\right)$ at the FC point, an effective population transfer to $\mathrm{G} \rightarrow \mathrm{C}(\mathrm{CT}) 1$ occurs. After $\sim 25 \mathrm{fs}$, the population of $\mathrm{G} \rightarrow \mathrm{C}(\mathrm{CT}) 1$ is 0.5 , and after $60 \mathrm{fs}$ it is 0.9 , and the transfer is virtually complete. No significant changes are then predicted until $250 \mathrm{fs}$, when the population of the other excited states is close to zero, the most populated one being $\mathrm{C}\left(\pi \pi^{*} 1\right)$. When exciting to $\mathrm{G}\left(\mathrm{L}_{\mathrm{b}}\right)$, an ultrafast decay to $G\left(L_{a}\right)$ is predicted, in line with the results obtained on $\mathrm{G}$ monomer. ${ }^{17}$ However, then $\mathrm{G}\left(\mathrm{L}_{\mathrm{a}}\right)$ acts as 'doorway' state to $\mathrm{G} \rightarrow \mathrm{C}(\mathrm{CT}) 1$, whose population after $\sim 60$ fs is
0.8. Interestingly, a small fraction of the population is 'trapped' on $\mathrm{G}\left(\mathrm{L}_{\mathrm{a}}\right)$ and then transferred to $\mathrm{C}\left(\pi \pi^{*} 1\right)$, whose population after 250 fs is non-negligible $(\sim 0.05)$.

When exciting to $\mathrm{C}\left(\pi \pi^{*} 1\right)$, the population is also effectively transferred to $\mathrm{G} \rightarrow \mathrm{C}(\mathrm{CT}) 1$, though the transfer is slower and less complete than when exciting the $\mathrm{G} \mathrm{L}_{\mathrm{a}} / \mathrm{L}_{\mathrm{b}}$ states. After $60 \mathrm{fs}$, the population of $\mathrm{C}\left(\pi \pi^{*} 1\right)$ is still 0.5 , and after 250 fs 0.15 . Finally, excitation of $\mathrm{C}\left(\pi \pi^{*} 2\right)$ triggers an ultrafast decay to $\mathrm{C}\left(\pi \pi^{*} 1\right)$ and, then, to $\mathrm{G} \rightarrow \mathrm{C}(\mathrm{CT}) 1$. In this case however, a plateau is reached after $\sim 100 \mathrm{fs}$, with $\mathrm{C}\left(\pi \pi^{*} 1\right)$ and $\mathrm{G} \rightarrow \mathrm{C}(\mathrm{CT}) 1$ exhibiting a similar population $(\sim 0.3$ and $\sim 0.5$, respectively).

The picture provided by $\omega \mathrm{B} 97 \mathrm{X}-\mathrm{D}$ is basically consistent with that of CAM-B3LYP. The $\sim 0.4 \mathrm{eV}$ larger energy of the $\mathrm{G} \rightarrow \mathrm{C}(\mathrm{CT}) 1$ state in the $\omega$ B97X-D model confers only a minor slow down of the population transfer, and after 100 fs the population of $\mathrm{G} \rightarrow \mathrm{C}(\mathrm{CT}) 1$ is $\geq 0.8$ when exciting $G\left(L_{a}\right)$ or $G\left(L_{b}\right)$, and $\geq 0.4$ when exciting $\mathrm{C}\left(\pi \pi^{*} 1\right)$ or $\mathrm{C}\left(\pi \pi^{*} 2\right)$. In this latter case the transfer to $\mathrm{G} \rightarrow \mathrm{C}(\mathrm{CT}) 1$ is actually even larger than according to CAM-B3LYP, and a plateau in the populations of $\mathrm{G} \rightarrow \mathrm{C}(\mathrm{CT}) 1$ and $\mathrm{C}\left(\pi \pi^{*} 1\right)$ is reached at a later stage, after $\sim 150$ fs.

Figure 3 also highlights that the population of the dark $n \pi^{*}$ excited states is extremely small, their sum being $\leq 0.02$ for all calculations except for an initial excitation to $\mathrm{C}\left(\pi \pi^{*} 2\right)$ where it is marginally larger $(\sim 0.06$ for the CAM-B3LYP and $\sim 0.04$ for the $\omega$ B97X-D models). WC pairing thus suppresses these deactivation channels, which are active in the cytosine monomer. ${ }^{15,17}$ Indeed, our recent QD studies on cytosine and 1-methylcytosine with a LVC Hamiltonian parametrized with CAMB3LYP predict that after initial photoexcitation of $\pi \pi^{*} 1 \sim 20-25 \%$ of the population is transferred to the $\mathrm{n} \pi^{*}$ states for cytosine and $\sim 10 \%$ for 1-methylcytosine, while exciting $\pi \pi^{*} 2$ the population transfer increases up to $\sim 50 \%$ for cytosine and $\sim 30 \%$ for 1-methylcytosine.

Given the small participation of the $\mathrm{n} \pi^{*}$ states and the $\mathrm{G} \rightarrow \mathrm{C}(\mathrm{CT}) 2$ state, it is unsurprising that the population dynamics predicted by the reduced-dimensionality 5-state model, includ- 


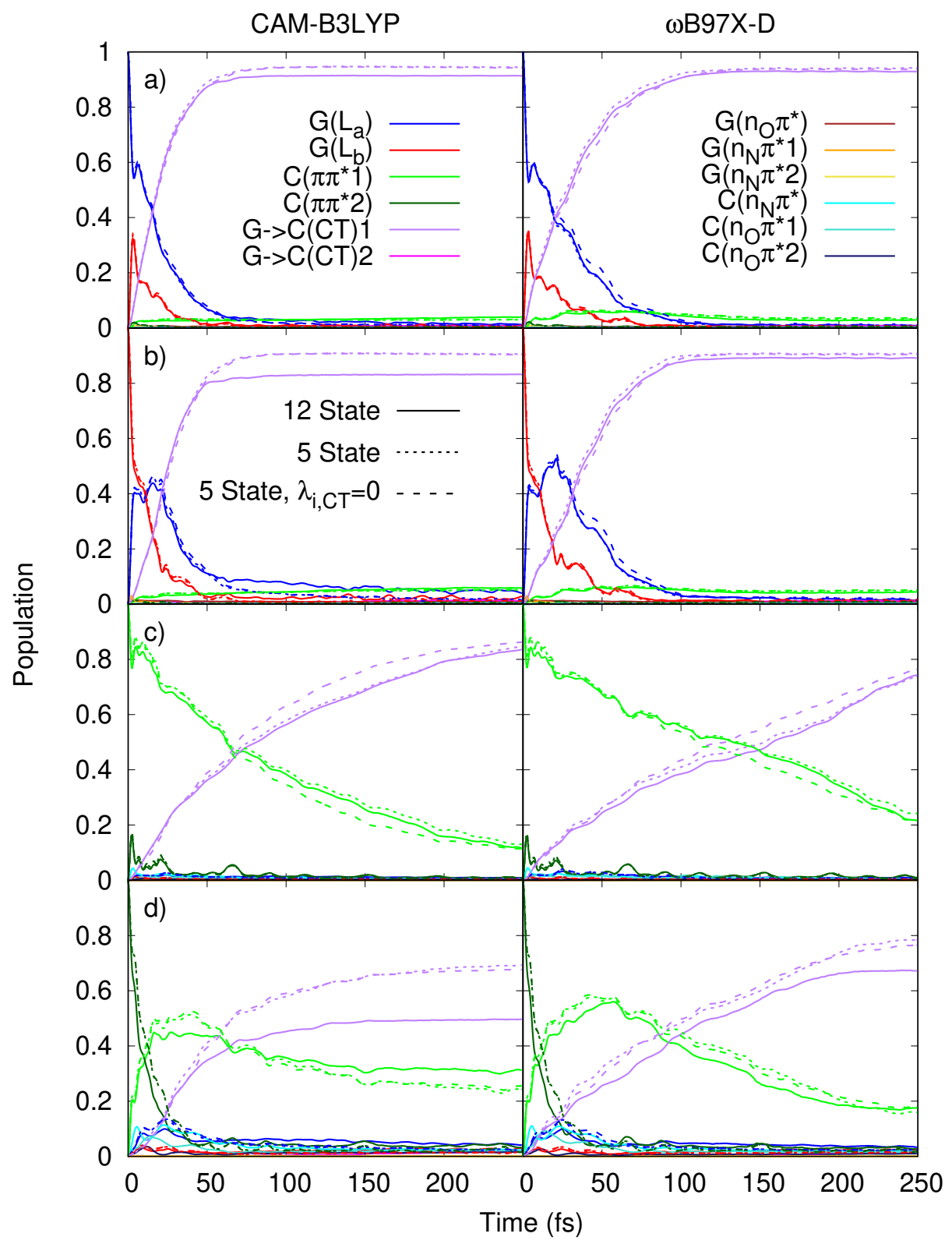

Figure 3: Diabatic state populations for FrD-LVC models with 12 states (solid line), 5 states (dotted line), and 5 states with $\boldsymbol{\lambda}_{i j}=0$ for all the $\pi \pi^{*}$ states coupled to $\mathrm{G} \rightarrow \mathrm{C}(\mathrm{CT}) 1$ (dashed line). Dynamics initiated on a) $\mathrm{G}\left(\mathrm{L}_{\mathrm{a}}\right)$, b) $\mathrm{G}\left(\mathrm{L}_{\mathrm{b}}\right)$, c) $\mathrm{C}\left(\pi \pi^{*} 1\right)$ and $\left.\mathrm{d}\right) \mathrm{C}\left(\pi \pi^{*} 2\right)$ states. Parametrized by CAM-B3LYP (left) and $\omega$ B97X-D (right).

ing only the $\pi \pi^{*}$ and $\mathrm{G} \rightarrow \mathrm{C}(\mathrm{CT}) 1$ states, and the 65 A' vibrational modes, are similar to that described above (dotted lines in Figure 3). The main difference is the 5 state model predicts a greater population transfer to the $\mathrm{G} \rightarrow \mathrm{C}(\mathrm{CT}) 1$ state and reduced 'trapping' in the $\mathrm{C}\left(\pi \pi^{*} 1\right)$ state after excitation to $\mathrm{C}\left(\pi \pi^{*} 2\right)$ in the CAMB3LYP model. Similar effects are seen, although to a lesser extent, in the $\omega$ B97X-D model, and also in the CAM-B3LYP model af- ter excitation to the $\mathrm{G}\left(\mathrm{L}_{\mathrm{b}}\right)$ state (where in this case there is less trapping in the $G\left(L_{a}\right)$ state). Whilst part of the less effective population of the $\mathrm{G} \rightarrow \mathrm{C}(\mathrm{CT}) 1$ state in the 12 state model can be attributed to the small population of the $\mathrm{n} \pi^{*}$ states, the remainder may be attributed to this trapping on the $\pi \pi^{*}$ states. 


\subsubsection{Vibrational effects}

In order to investigate the cause of the 'trapping' in the 12 state model, we have analysed the expectation values of the diabatic potentials of the different states, integrating over all normal modes of the wavepacket as a function of time in Figure. 4. For clarity, this figure only shows the potentials of the $\pi \pi^{*}$ and $n \pi^{*}$ states on the base that is initially excited, as well as the $\mathrm{G} \rightarrow \mathrm{C}(\mathrm{CT}) 1$ state. The full figure may be found in the SI, Figure S9.

For initial excitation to $\mathrm{C}\left(\pi \pi^{*} 2\right)$ in the CAMB3LYP model, where the trapping effect is most clearly observed, the left panel of Figure $4 \mathrm{~d}$ shows that the potential of $\mathrm{C}\left(\pi \pi^{*} 1\right)$ in the 12 state model almost plateaus after $\sim 50 \mathrm{fs}$,

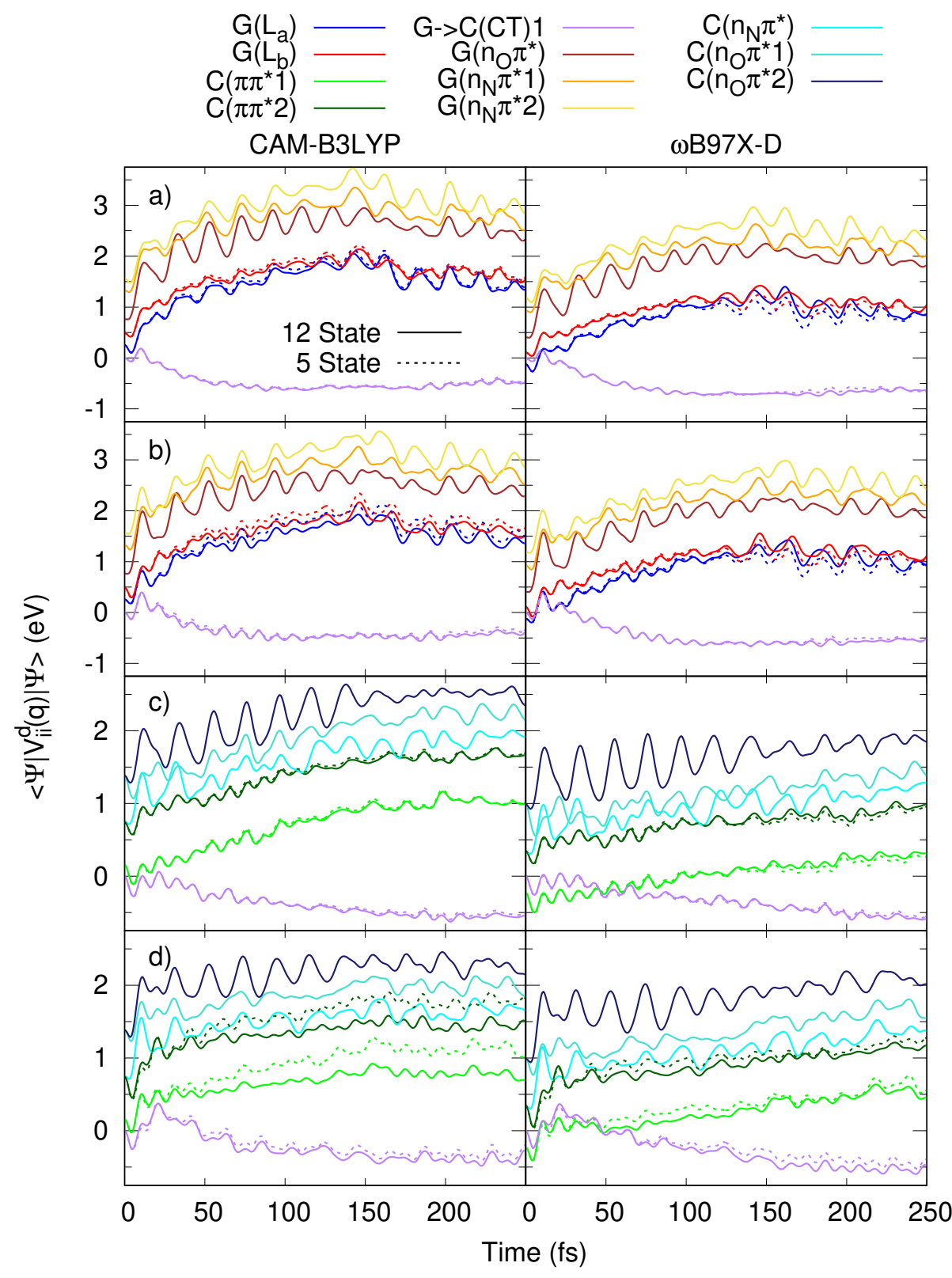

Figure 4: Expectation of diabatic state potential energies, $\left(\left\langle\Psi\left|V_{i i}^{\mathrm{d}}(\boldsymbol{q})\right| \Psi\right\rangle\right)$ shifted by $-E_{\mathrm{CT} 1}^{\mathrm{d}}(0)-$ $1 / 4 \sum_{\alpha} \omega_{\alpha}$ so that the $\mathrm{G} \rightarrow \mathrm{C}(\mathrm{CT}) 1$ energy is 0 initially. For the 12 state (solid lines) and 5 state (dotted lines) FrD-LVC models for GC, parametrized by left: CAM-B3LYP and right: $\omega$ B97X-D for dynamics initiated on a) $\mathrm{G}\left(\mathrm{L}_{\mathrm{a}}\right)$, b) $\mathrm{G}\left(\mathrm{L}_{\mathrm{b}}\right)$, c) $\mathrm{C}\left(\pi \pi^{*} 1\right)$ and d) $\mathrm{C}\left(\pi \pi^{*} 2\right)$ states. For clarity, only the potentials of the $\mathrm{G} \rightarrow \mathrm{C}(\mathrm{CT}) 1$ state, and the $\pi \pi^{*}$ and $\mathrm{n} \pi^{*}$ states localized on the base that is initially excited are shown. 
whereas in the 5 state model it is destabilised. Many vibrations are slightly more active in the 5 state than the 12 state model, resulting in this destabilisation of $\mathrm{C}\left(\pi \pi^{*} 1\right)$ and, therefore, to a larger transfer to the $\mathrm{G} \rightarrow \mathrm{C}(\mathrm{CT}) 1$ state for the 5 state model. The expectation of the $\mathrm{C}\left(\pi \pi^{*} 1\right)$ diabatic potential along a representative few normal modes is shown in Figure 5, with the displacement vectors of the vibrations illustrated in the SI Figure S6. In the 12 state model there is a larger bath of vibrational modes where the excess energy deposited when exciting $\mathrm{C}\left(\pi \pi^{*} 2\right)$ can be dissipated (as the 12 state model includes all 99 A' and A" modes, whereas the 5 state model includes only the 65 A' modes), cooling down the vibrations that destabilize the $\mathrm{C}\left(\pi \pi^{*} 1\right)$ state. In contrast, cooling down these vibrations does not affect the $\mathrm{G} \rightarrow \mathrm{C}(\mathrm{CT}) 1$ state as significantly. This explanation correlates with the fact that the energy gap between initial excitation and $\mathrm{G} \rightarrow \mathrm{C}(\mathrm{CT}) 1$ state is largest for initial excitation to $\mathrm{C}\left(\pi \pi^{*} 2\right)$ in the CAM-B3LYP model, hence this is the case where the largest amount of energy has to be dissipated in the vibrational modes in the subsequent reorganisation, and where the largest trapping is observed.

Figure. 4 also reveals that the $\mathrm{n} \pi^{*}$ states are immediately destabilised upon excitation, within $\sim 10$ fs the diabatic potential of the lowest $\mathrm{n} \pi^{*}$ state on each base reaches a value $>0.5 \mathrm{eV}$ larger than at the FC point. In particular, when the initial excitation is close in energy to the $n \pi^{*}$ state, so to either $G\left(L_{b}\right)$ or $\mathrm{C}\left(\pi \pi^{*} 2\right)$, the $\pi \pi^{*}$ state of initial excitation is immediately stabilised and the closest $\mathrm{n} \pi^{*}$ state destabilised. This helps to explain why there is only small population transfer to the $\mathrm{n} \pi^{*}$ states, as by the time the vibrational motions bring the $\mathrm{G}\left(\mathrm{L}_{\mathrm{b}}\right) / \mathrm{G}\left(\mathrm{n}_{\mathrm{O}} \pi^{*}\right)$ or $\mathrm{C}\left(\pi \pi^{*} 2\right) / \mathrm{C}\left(\mathrm{n}_{\mathrm{N}} \pi^{*}\right)$ states close in energy once more, after $\sim 20 \mathrm{fs}$, the population has already been deposited to the lower $\mathrm{G}\left(\mathrm{L}_{\mathrm{a}}\right)$ or $\mathrm{C}\left(\pi \pi^{*} 1\right)$ states and subsequently to the $\mathrm{G} \rightarrow \mathrm{C}(\mathrm{CT}) 1$.

Whilst this is very much a multi-vibrational mode effect, we have identified two normal modes that provide a large contribution to this process, $q_{76}$ for the $\mathrm{G}\left(\mathrm{L}_{\mathrm{b}}\right)-\mathrm{G}\left(\mathrm{n}_{\mathrm{O}} \pi^{*}\right)$ gap, and $q_{77}$ for the $\mathrm{C}\left(\pi \pi^{*} 2\right)-\mathrm{C}\left(\mathrm{n}_{\mathrm{N}} \pi^{*}\right)$ gap. The $q_{76}$ mode is predominantly localized on $\mathrm{G}$, and consists of an N1-H1 and C8-H8 bending motion, whilst $q_{77}$ is predominantly localized on $\mathrm{C}$, and consti-

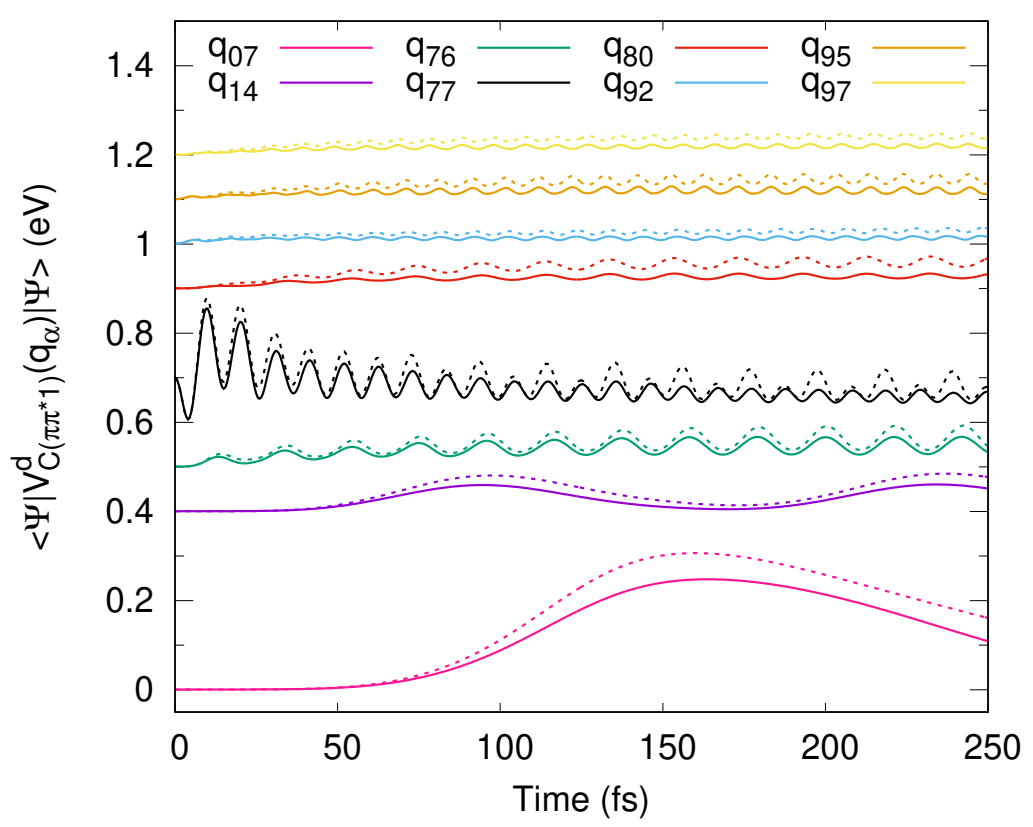

Figure 5: Expectation of diabatic state potential energy along various normal modes for the $\mathrm{C}\left(\pi \pi^{*} 1\right)$ state $\left(\left\langle\Psi\left|V_{\mathrm{C}\left(\pi \pi^{*}\right)}^{\mathrm{d}}\left(q_{\alpha}\right)\right| \Psi\right\rangle\right)$, after initial excitation to the $\mathrm{C}\left(\pi \pi^{*} 2\right)$ state for the 12 state (solid lines) and 5 state (dotted lines) CAM-B3LYP parametrized FrD-LVC models. Each mode offset by different values so that they do not overlap. 
tutes a combined $\mathrm{NH}_{2}$ bend, C4-C5 ring stretch and $\mathrm{C} 5-\mathrm{H} 5$ and $\mathrm{C} 6-\mathrm{H} 6$ bend. The expectation of the diabatic potentials along the $q_{76}$ mode after initial excitation to $\mathrm{G}\left(\mathrm{L}_{\mathrm{b}}\right)$, and along the $q_{77}$ mode after initial excitation to $\mathrm{C}\left(\pi \pi^{*} 2\right)$ are shown in Figure 6, panels a) and b) respectively, for the first 25 fs of the calculation with the $\omega$ B97X-D FrD-LVC model. In this Figure we observe, indeed, that after initial excitation, the $\mathrm{G}\left(\mathrm{L}_{\mathrm{b}}\right) / \mathrm{C}\left(\pi \pi^{*} 2\right)$ states are immediately stabilised, whilst the $\mathrm{G}\left(\mathrm{n}_{\mathrm{O}} \pi^{*}\right) / \mathrm{C}\left(\mathrm{n}_{\mathrm{N}} \pi^{*}\right)$ states are destabilised within half a vibrational period ( $\sim 10 \mathrm{fs})$. After the vibrational period is complete $(\sim 20 \mathrm{fs})$ and the $\pi \pi^{*}$ and $\mathrm{n} \pi^{*}$ states are close in energy once more, the population from the $\mathrm{G}\left(\mathrm{L}_{\mathrm{b}}\right) / \mathrm{C}\left(\pi \pi^{*} 2\right)$ states is already close to zero (dotted lines in Figure 6).

In the SI we show that the results for the CAM-B3LYP model are the same, the effect of these modes after initial excitation to the other states, and their value for the full 250 fs of the propagation (Figures S10 and S11). Interestingly the mode $q_{77}$, despite being localized on $\mathrm{C}$, significantly affects the states localized on $\mathrm{G}$.

Another interesting vibrational effect is the large reorganisation energy of the $\mathrm{G} \rightarrow \mathrm{C}(\mathrm{CT}) 1$ state after it is populated, leaving it $\sim 0.5 \mathrm{eV}$ more stable after 250 fs than at the FC point, whilst the $\pi \pi^{*}$ states are $>0.5 \mathrm{eV}$ destabilised, leaving the gap between the $\mathrm{G} \rightarrow \mathrm{C}(\mathrm{CT}) 1$ and lowest $\pi \pi^{*}$ state $>1 \mathrm{eV}$ (Figure 4 ). The $q_{76}$ and $q_{77}$ modes previously mentioned contribute
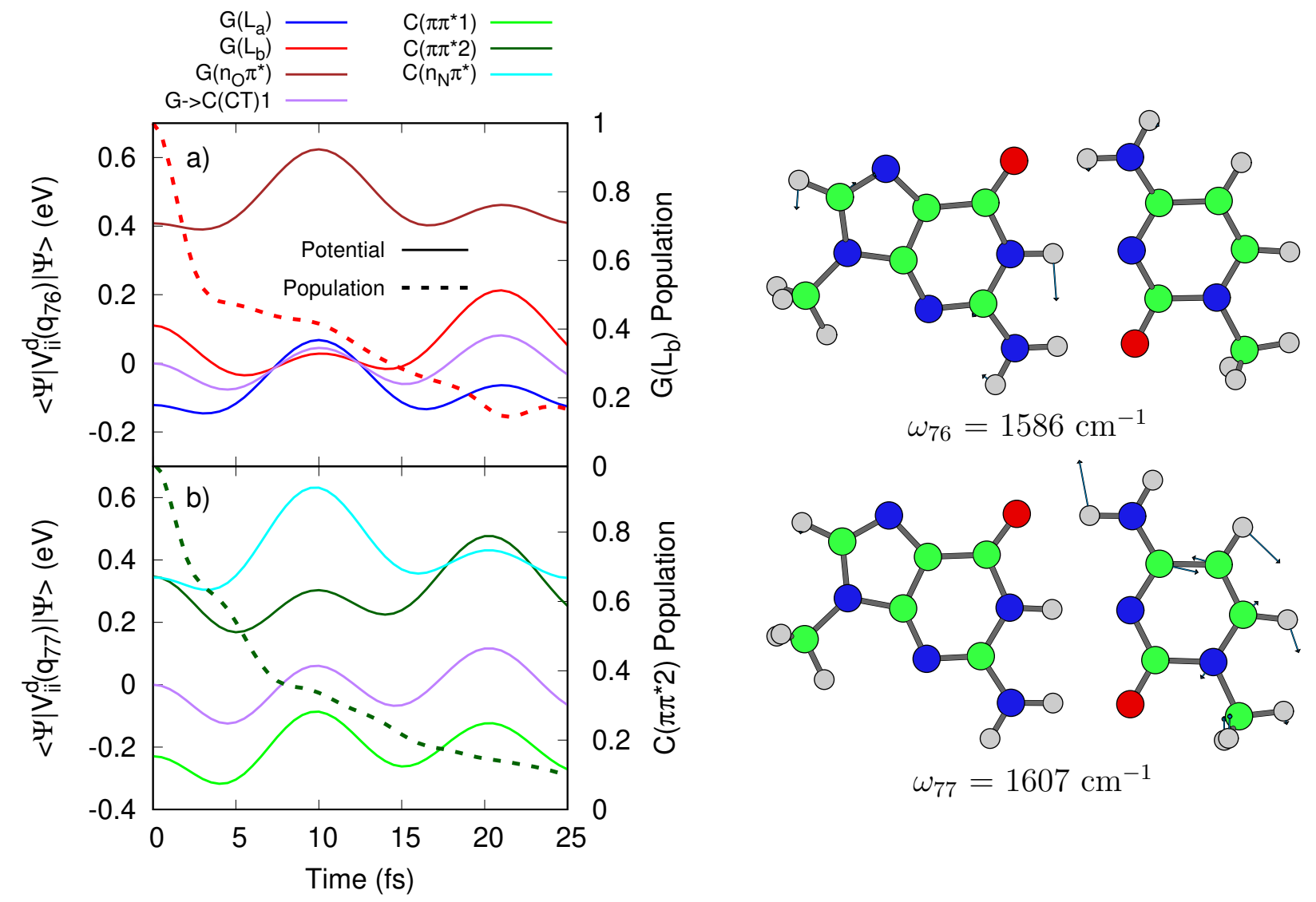

Figure 6: Left: Expectation of diabatic state potential energies (solid lines) along the a) $q_{76}$ normal mode after initial excitation to $\mathrm{G}\left(\mathrm{L}_{\mathrm{b}}\right)$ and $\left.\mathrm{b}\right) q_{77}$ normal mode after initial excitation to $\mathrm{C}\left(\pi \pi^{*} 2\right)$. Calculated with the 12 state FrD-LVC model for GC parametrized by $\omega$ B97X-D. For clarity the potentials are shifted so that the $\mathrm{G} \rightarrow \mathrm{C}(\mathrm{CT}) 1$ energy is 0 initially, i.e. by $-E_{\mathrm{CT} 1}^{\mathrm{d}}(0)-1 / 4 \omega_{76 / 77}$, and only the potentials of the $\mathrm{G} \rightarrow \mathrm{C}(\mathrm{CT}) 1$ state, and the $\pi \pi^{*}$ and lowest $\mathrm{n} \pi^{*}$ states localized on the base that is initially excited are shown. The dotted lines show the population of the $G\left(\mathrm{~L}_{\mathrm{b}}\right)$ state in a) and the $\mathrm{C}\left(\pi \pi^{*} 2\right)$ state in b). On the right hand side are the displacement vectors of the $q_{76}$ and $q_{77}$ normal modes and their frequencies. 
somewhat to this effect (see SI, Figures S10 and S11), however a much larger contribution arises from a low frequency mode $q_{07}$, that involves a shearing motion between the bases. An example of its affect on the expectation of the diabatic potential energies following initial excitation to a) $\mathrm{G}\left(\mathrm{L}_{\mathrm{a}}\right)$ and b) $\mathrm{C}\left(\pi \pi^{*} 1\right)$ is shown in Figure 7 for the $\omega$ B97X-D model. It is not active for the first 50 fs of the calculation, however when the $\mathrm{G} \rightarrow \mathrm{C}(\mathrm{CT}) 1$ state is significantly populated it becomes active and stabilises the $\mathrm{G} \rightarrow \mathrm{C}(\mathrm{CT}) 1$ state, whilst destabilising the others. The effect of this mode on the diabatic potentials following excitations to the other $\pi \pi^{*}$ states is shown in the SI, Figure S12 along with the CAM-B3LYP results, and the effect is much the same.

The impact of the vibrational motion on

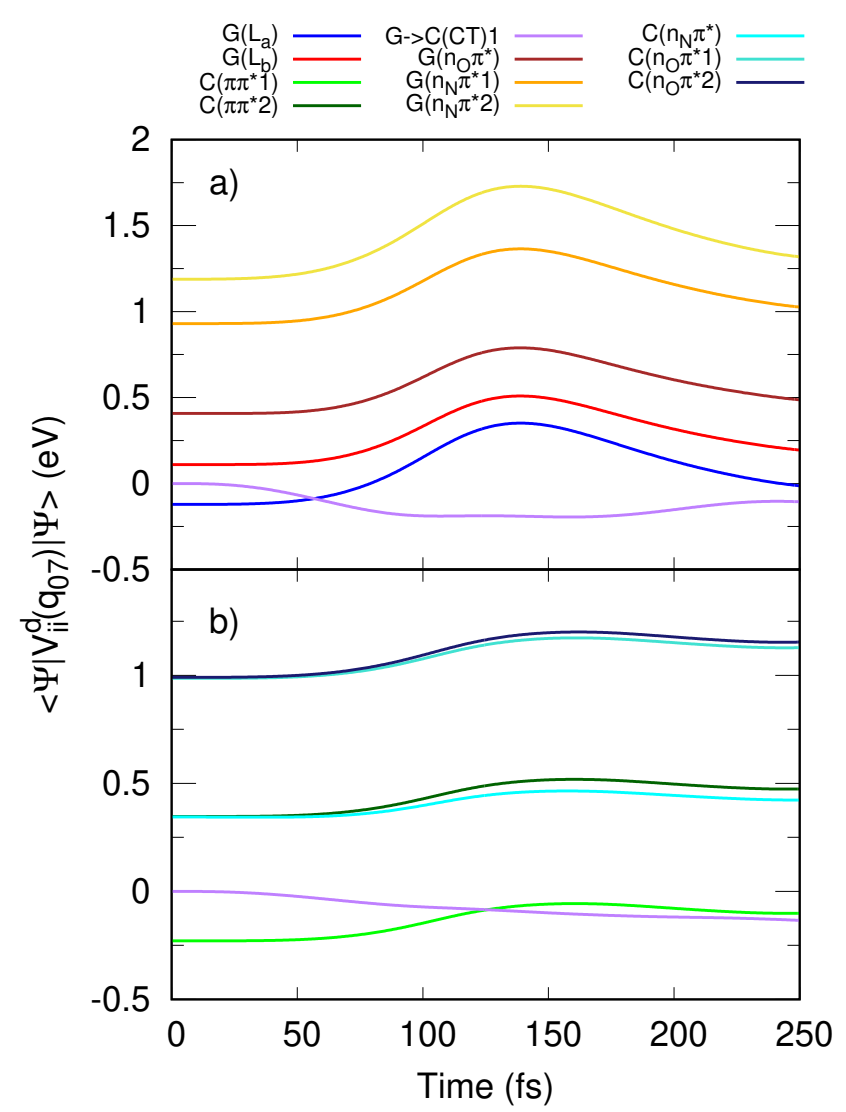

the diabatic coupling terms (i.e. $\left.V_{i j}^{\mathrm{d}}(\boldsymbol{q})\right)$ between the $\pi \pi^{*}$ and $\mathrm{G} \rightarrow \mathrm{C}(\mathrm{CT}) 1$ states is instead more limited. In particular, Figure S13 in the SI shows after an excitation to $G\left(L_{a}\right)$ or $\mathrm{G}\left(\mathrm{L}_{\mathrm{b}}\right)$ the coupling between the doorway state $\mathrm{G}\left(\mathrm{L}_{\mathrm{a}}\right)$ and the $\mathrm{G} \rightarrow \mathrm{C}(\mathrm{CT}) 1$ does not significantly deviate from its initial value at the FC point (Table 2). An analogously weak time-dependence is observed for the coupling of $\mathrm{C}\left(\pi \pi^{*} 1\right)$ with $\mathrm{G} \rightarrow \mathrm{C}(\mathrm{CT}) 1$ after an initial excitation to $\mathrm{C}\left(\pi \pi^{*} 1\right)$ and $\mathrm{C}\left(\pi \pi^{*} 2\right)$. As a consequence, the dashed lines Figure. 3 show that neglecting the dependence of $V_{i j}^{\mathrm{d}}(\boldsymbol{q})$ on coordinate for the $\pi \pi^{*}-\mathrm{G} \rightarrow \mathrm{C}(\mathrm{CT}) 1$ couplings (i.e. setting the linear terms $\boldsymbol{\lambda}_{i j}=0$ for $\left.i, j=\mathrm{G} \rightarrow \mathrm{C}(\mathrm{CT}) 1\right)$ produces no discernible difference to the dynamics of the 5 state FrD-LVC models.

The excitonic couplings, i.e. those between

Figure 7: Left: Expectation of diabatic state potential energies along the $q_{07}$ normal mode (i.e. $\left.\left\langle\Psi\left|V_{i i}^{\mathrm{d}}\left(q_{07}\right)\right| \Psi\right\rangle\right)$ from the 12 state FrD-LVC model for GC parametrized by $\omega$ B97X-D after initial excitation to a) $\mathrm{G}\left(\mathrm{L}_{\mathrm{a}}\right)$ and b) $\mathrm{C}\left(\pi \pi^{*} 1\right)$. For clarity the potentials are shifted so that the $\mathrm{G} \rightarrow \mathrm{C}(\mathrm{CT}) 1$ energy is 0 initially, i.e. by $-E_{\mathrm{CT} 1}^{\mathrm{d}}(0)-1 / 4 \omega_{07}$, and only the potentials of the $\mathrm{G} \rightarrow \mathrm{C}(\mathrm{CT}) 1$ state, and the $\pi \pi^{*}$ and $\mathrm{n} \pi^{*}$ states localized on the base that is initially excited are shown. On the right hand side are the displacement vectors of the $q_{07}$ normal mode and its vibrational frequency. 
$\pi \pi^{*}$ states localized on each base, are similarly unaffected by the vibrations, see Figure S14 in the SI. In particular, the excitonic coupling between the doorway states, $\mathrm{G}\left(\mathrm{L}_{\mathrm{a}}\right)-\mathrm{C}\left(\pi \pi^{*} 1\right)$, remains very small during the propagation, explaining the limited population transfer from states localized on one base to ones localized on the other.

On the contrary, the couplings between the bright states localized on the same base are strongly affected by vibrational motions, see Figure S15 in the SI. A tangible consequence, for example, is that the strong mixing of $G\left(L_{a}\right)$ $\mathrm{G}\left(\mathrm{L}_{\mathrm{b}}\right)$ and $\mathrm{C}\left(\pi \pi^{*} 1\right)-\mathrm{C}\left(\pi \pi^{*} 2\right)$ states observed in the adiabatic states at the $\mathrm{FC}$ point decreases during the propagation, such that the adiabatic states end up resembling much more the reference states on isolated monomers.

Finally, we have analysed how the excited state dynamics is affected by deuteration, i.e. the substitution of the five 'exchangeable' protons (the amino and imino ones) by deuterium. Many TR studies on DNA are performed in deuterated solvents, ${ }^{50}$ and isotope substitution has been suggested to affect the PCET processes. ${ }^{74}$ As shown in Figure S7 of the SI the population dynamics is very little affected by deuteration, which decreases the transfer to $\mathrm{G} \rightarrow \mathrm{C}(\mathrm{CT}) 1$ by $\leq 5 \%$. As a consequence the first step of the PCET reaction is not expected to be very sensitive to isotope effect, at least in the gas phase.

\subsubsection{GC absorption spectrum}

We have also computed the absorption spectrum of the GC pair, based on our 12 state FrD-LVC model calculations, using the procedure described in the Computational Details, and SI, Section S1.2. These are shown in Figure 8, with panel a) illustrating the individ-

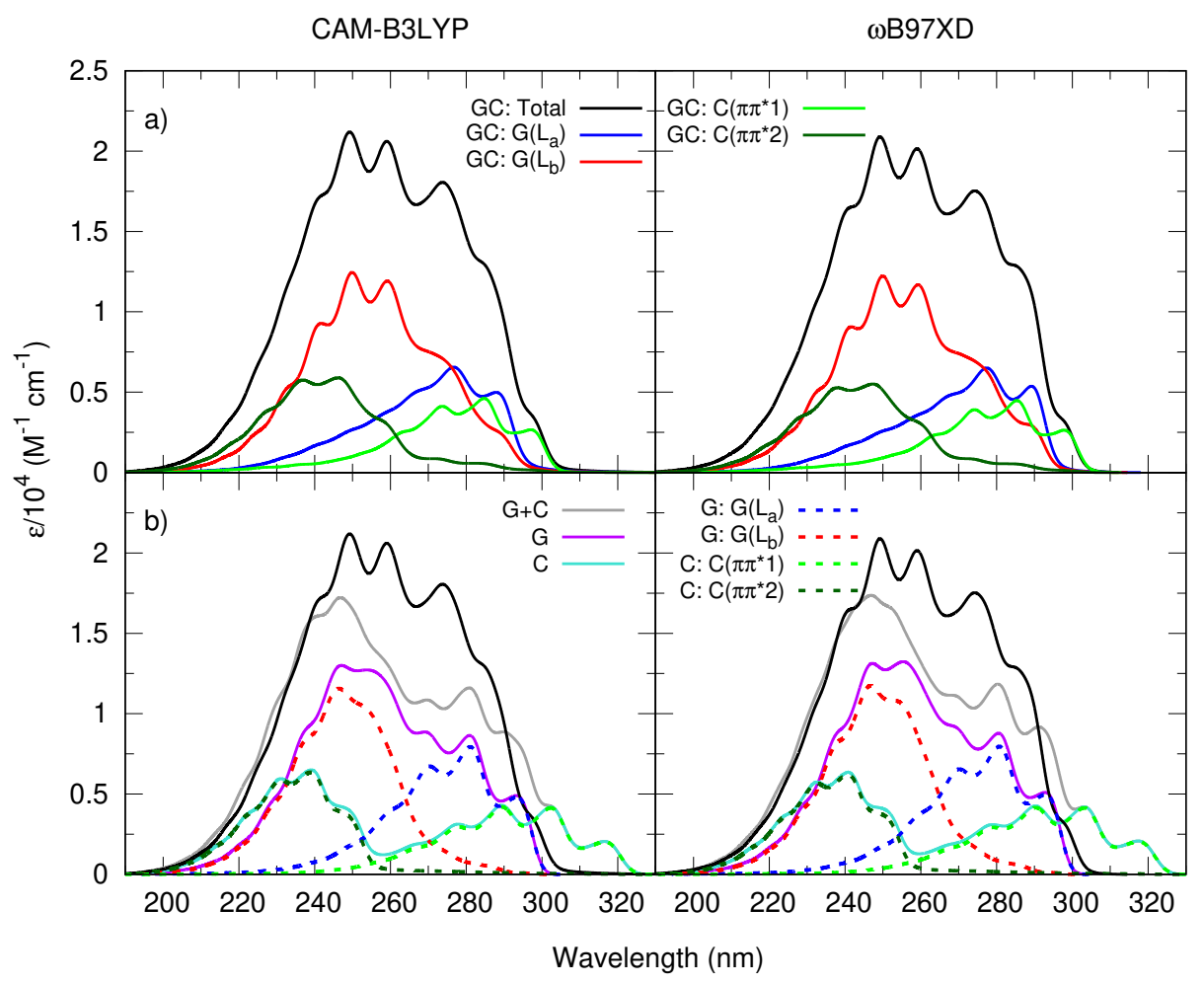

Figure 8: Absorption spectra of GC calculated from the 12 state FrD-LVC models (solid black line) with a) individual diabatic state components (solid blue, red, light and dark green lines) and b) compared to monomeric spectra of $\mathrm{G}$ (purple) and $\mathrm{C}$ (turquoise) with individual state components (dashed red, blue, light and dark green lines) and the sum of $\mathrm{G}$ and $\mathrm{C}$ (grey). LVC models parametrized by CAM-B3LYP (left) and $\omega$ B97X-D (right). All spectra shifted by $-0.75 \mathrm{eV}$ and broadened with Gaussian HWHM=0.04 eV. 
ual state components of the GC spectrum, and panel b) comparing it to absorption spectra of isolated $\mathrm{G}$ and $\mathrm{C}$ and their sum. The absorption spectra of isolated $\mathrm{G}$ and $\mathrm{C}$ are calculated using 5 state LVC models, including $2 \pi \pi^{*}$ and $3 \mathrm{n} \pi^{*}$ states for each base, in the same manner as our recent work on single nucleobases. ${ }^{17}$ All spectra are shifted by $-0.75 \mathrm{eV}$ to match the onset of the GC spectrum in chloroform, ${ }^{42}$ (see Figure S18 in SI) and plotted on the wavelength scale. The parametrizations with CAMB3LYP and $\omega$ B97X-D predicted quite similar spectra, therefore our analysis below applies to both models.

Figure $8 \mathrm{a}$ shows that the region of the maximum absorption at $\sim 250-260 \mathrm{~nm}$ is dominated by the contribution of the $G\left(L_{b}\right)$. At shorter wavelengths it is reinforced and then slightly overcome by the contribution of $\mathrm{C}\left(\pi \pi^{*} 2\right)$. Whilst on the red-wing, at longer wavelengths, most of the signal arises from $\mathrm{G}\left(\mathrm{L}_{\mathrm{a}}\right)$ and $\mathrm{C}\left(\pi \pi^{*} 1\right)$. In this red wing, according to our computations most of the wavepacket should be located on $\mathrm{G}$ in the $\sim 280-295 \mathrm{~nm}$ window, whilst the contribution from $\mathrm{C}\left(\pi \pi^{*} 1\right)$ is only predominant at $\geq 295 \mathrm{~nm}$.

Comparison with the results in Figure $8 \mathrm{~b}$ indicate that the contribution of each individual state is broader in GC than the individual monomers, due to the large $\pi \pi^{*}$ mixing couplings and coupling to the $\mathrm{G} \rightarrow \mathrm{C}(\mathrm{CT}) 1$ state in GC. Furthermore, the GC spectrum exhibits interesting differences with respect to the sum of monomer spectrum $(\mathrm{G}+\mathrm{C})$. First, the $\mathrm{G}+\mathrm{C}$ spectrum exhibits a longer red wing tail, coming from the $\mathrm{C}$ contribution, which disappears in GC. This outcome is due to blue shifting of $\mathrm{C}\left(\pi \pi^{*} 1\right)$ due to hydrogen bonding. ${ }^{75}$ Second, the maximum of the absorption band of $\mathrm{GC}$ is weakly red-shifted with respect to $\mathrm{G}+\mathrm{C}$ one and it is more intense in the region 260-290 nm. A similar effect is observed, although to a lesser extent, in experiment. ${ }^{42}$ Here we assign it partially to the blue shifting of $\mathrm{C}\left(\pi \pi^{*} 1\right)$, and partially due to weak red-shifting of the $\mathrm{G}\left(\mathrm{L}_{\mathrm{b}}\right)$ contribution.

\section{Concluding remarks}

In this contribution we have introduced a method for an automatic parametrization of a generalised LVC Hamiltonian suitable for studying the photophysics of multichromophoric assemblies, exploiting TD-DFT. Its main methodological novelty concerns the possibility of defining the reference diabatic states on the basis of isolated fragments. In combination with effective wavepacket propagations, for instance with the ML-MCTDH method, our approach makes it rather straightforward to describe the competition between inter- and intrachromophore photophysical pathways within a fully quantum dynamical approach. This method is thus an attractive option to study phenomena where the timescale of internal conversion processes on individual chromophores is expected to be similar to that of energy and charge transfer between multiple chromophores. Furthermore, it can also account for the possible coupling between these processes.

Due to the efficiency of the parametrization of the LVC Hamiltonian and capability of ML-MCTDH propagations, it is possible to include many electronic states $(>10)$ and dozen/hundreds of normal coordinates in the model. This permits the investigation of the ultrafast vibronic dynamics following a photoexcitation in significantly broad energy ranges. For example, in the present study we focused on the region above $\sim 220 \mathrm{~nm}$, i.e. that 'covered' by the four lowest energy bright states of a GC pair. However, we have simulated the dynamics considering up to 12 excited states (i.e. including also the lowest energy dark states). This is typically more than can be considered with 'on-the-fly' dynamics calculations, such as surface hopping (although, one should remember that the number of adiabatic states to describe the same dynamics could be smaller). ${ }^{76}$ Therefore, even in cases where an LVC Hamiltonian cannot capture all the details of the adiabatic PES explored with surface hopping methods, this approach could complement these calculations by providing a relatively quick assessment of the role of higher excited states and/or the role quantum dynamics effects. Indeed, the pro- 
posed parametrization is quite effective: TDDFT calculations for the diabatization with 6$31 \mathrm{G}(\mathrm{d})$ basis set in the present work took only $\sim 24$ hours using 28 cores, whilst the diabatization took $\sim 30$ minutes on 12 cores. For the quantum dynamics calculations themselves the computation times ranged between 100-300 hours on a single core for the 12 state calculations, and 20-100 hours for the 5 state calculations. These values could be feasibly reduced through appropriate tuning of parameters in the ML-MCTDH computations, such as choice of integrator, mode combination, and multilayer 'tree', or by discarding smaller terms in the FrD-LVC Hamiltonian.

The application of FrD-LVC is most suited when couplings between the different monomers are not excessively strong, and more in general when it is conceivable or interesting to hypothesize to have prepared an initial state localized on one single monomer. In those cases, the proposed approach also has the advantage to be naturally allied with the chemical point of view that breaks the investigated photophysical event into elementary processes on the individual molecular components and their possible interactions. For example, this is typically done, more or less implicitly, for charge separation/migration/recombination in multichromophore assemblies.

The application of FrD-LVC should be handled with more care when the coupling between the different units is so strong to make any connection with the behavior of the separated unit too weak. The analysis of the results of the fragment diabatization procedure, such as calculated couplings and predicted minima, and/or of the subsequent dynamics can provide useful diagnostics to identify these 'problematic' cases. However, it is important to stress that FrD-LVC could still be used even in these cases by considering initial states coherently excited on more than one diabatic state or, alternatively, performing propagations by explicitly including the interaction with the pump laser pulse in the dynamics.

Our approach also obviously suffers from all the limitations of LVC models, such as lack of large amplitude motions, Duschinsky mix- ings and anharmonicity. Several strategies may be employed to ameliorate these deficiencies, such as moving to a quadratic vibronic coupling model (or at least some quadratic corrections like done in Refs. 16,71,77), fitting with non-harmonic functions when anharmonicity is confined to a few degrees of freedom, and mixed quantum classical approaches to deal with large amplitude motions. ${ }^{78}$ Such developments fall, however, outside the aim of the present contribution and will be tackled in future studies.

In the present, first, application of FrD-LVC we have studied the excited state dynamics of GC adopting a WC hydrogen bond arrangement, after exciting each of the four lowest energy bright states. We predict a substantial and fast population transfer from the initially excited bright state towards the lowest energy $\mathrm{G} \rightarrow \mathrm{C}$ CT state. This transfer is more effective for initial excitations on $\mathrm{G}$ bright states, with $\sim 80 \%$ of the population transferred in $\sim 50$ fs. However, also when exciting $\mathrm{C}\left(\pi \pi^{*} 1\right)$ and $\mathrm{C}\left(\pi \pi^{*} 2\right)$, in less than 100 fs $\sim 50 \%$ of the population is transferred to the $\mathrm{G} \rightarrow \mathrm{C}(\mathrm{CT}) 1$ state. Therefore, the fact that our Hamiltonian does not include the monomer $\mathrm{G}\left(\mathrm{L}_{\mathrm{a}}\right)$ and $\mathrm{C}\left(\pi \pi^{*} 1\right)$ decay channels to $\mathrm{S}_{0}$, involving large out-of-plane distortions, ${ }^{1}$ does not bias the qualitative reliability of our conclusion, given that the transfer to the $\mathrm{CT}$ states occurs on a time-scale $(<100 \mathrm{fs})$ for which the decay to $\mathrm{S}_{0}$ of the bright states should play a minor role.

Our dynamical calculations are thus consistent with the experimental picture in the gas phase, ${ }^{52}$ with the population of the bright excited states disappearing on a $<100$ fs time scale. Considering that on the CT PES the proton transfer is barrierless, our study also seems to support the reliability of the mechanism proposed by Sobolweski and Domcke . ${ }^{32,33}$ A forthcoming work will be devoted to study in depth the quantum dynamics of the coupling between the CT formation and the PT process.

FrD-LVC provides interesting insights on the effects ruling the yield of the CT population. For example, $\mathrm{G}\left(\mathrm{L}_{\mathrm{a}}\right)$ appears to be the excited state most strongly coupled to $\mathrm{G} \rightarrow \mathrm{C}(\mathrm{CT}) 1$, and permits the fastest and most effective popula- 
tion of $\mathrm{G} \rightarrow \mathrm{C}(\mathrm{CT}) 1$. This is true even when the $\mathrm{G} \rightarrow \mathrm{C}(\mathrm{CT}) 1$ state is closer in energy to other $\pi \pi^{*}$ states, such as predicted with the $\omega \mathrm{B} 97 \mathrm{X}$ $\mathrm{D}$ functional. Moreover, the extremely fast $\mathrm{G}\left(\mathrm{L}_{\mathrm{b}}\right) \rightarrow \mathrm{G}\left(\mathrm{L}_{\mathrm{a}}\right)$ decay suggests that $\mathrm{G}\left(\mathrm{L}_{\mathrm{a}}\right)$ is the 'main' doorway state to $\mathrm{G} \rightarrow \mathrm{C}(\mathrm{CT}) 1$.

Despite the smaller coupling of the $\mathrm{C} \pi \pi^{*}$ states to the $\mathrm{G} \rightarrow \mathrm{C}(\mathrm{CT}) 1$ state, we still observe effective (albeit slightly slower) population transfer when the initial excitation is localized on $\mathrm{C}$. The extremely fast $\mathrm{C}\left(\pi \pi^{*} 2\right) \rightarrow \mathrm{C}\left(\pi \pi^{*} 1\right)$ decay, combined with the small $\mathrm{C}\left(\pi \pi^{*} 1\right)-\mathrm{G}\left(\mathrm{L}_{\mathrm{a}}\right)$ excitonic coupling indicates that $\mathrm{C}\left(\pi \pi^{*} 1\right)$ acts as a secondary doorway state to $\mathrm{G} \rightarrow \mathrm{C}(\mathrm{CT}) 1$. These evidences lead us to conclude that a dramatic dependence of the CT process on the excitation wavelength should not be expected.

Interestingly, vibrational motions play only minor role in modulating the coupling between local excitations on different chromophores and with CT states. This does not mean, however, that vibrations do not drastically affect the yield of the population transfer, since they determine the reorganization energy of any excited state and, therefore, the relative stability of their minima. In Section S4.4 in the SI, we show that a trivial purely electronic dynamics with nuclei frozen at the FC position would provide a much smaller average population of $\mathrm{G} \rightarrow \mathrm{C}(\mathrm{CT}) 1$, even when it is the lowest energy excited state in the FC region. Furthermore, the purely electronic dynamics with the $\omega$ B97X-D model also predicts a significant excitonic transfer between the doorway $G\left(L_{a}\right)$ and $\mathrm{C}\left(\pi \pi^{*} 1\right)$ states, in contrast with the vibronic dynamics in Figure 3.

Our calculations indicate that the large reorganisation energy of the $\mathrm{G} \rightarrow \mathrm{C}(\mathrm{CT}) 1$ is the key to explain its population. As shown in Table 3, the predicted planar adiabatic and diabatic minima of the $\mathrm{G} \rightarrow \mathrm{C}(\mathrm{CT}) 1$ state are $\sim 1 \mathrm{eV}$ more stable than those of the $\pi \pi^{*}$ states according to CAM-B3LYP, and $\sim 0.6 \mathrm{eV}$ more stable according to $\omega \mathrm{B} 97 \mathrm{X}-\mathrm{D}$. This stability results in similar population transfer to the $\mathrm{G} \rightarrow \mathrm{C}(\mathrm{CT}) 1$ state by both CAM-B3LYP and $\omega$ B97X-D models, despite the $\mathrm{G} \rightarrow \mathrm{C}(\mathrm{CT}) 1$ state not being the lowest lying state at the $\mathrm{FC}$ point with $\omega$ B97X-D, and it lying $0.4 \mathrm{eV}$ higher in en- ergy than with CAM-B3LYP. Numerical experiments in the SI Section S4.5 indicate that, in order to overcome this effect and make population transfer to $\mathrm{G} \rightarrow \mathrm{C}(\mathrm{CT}) 1$ significantly smaller, the diabatic energy gap between the CT state and doorway state should be $\geq 0.6 \mathrm{eV}$.

WC pairing, besides allowing the formation of the CT states, has another important effect on the photoactivated dynamics of $\mathrm{G}$ and $\mathrm{C}$, almost suppressing any population transfer to the dark $\mathrm{n} \pi^{*}$ states. This internal conversion route is significant for isolated bases in the gas phase, in particular for $\mathrm{C}$ in the context of the present work. ${ }^{14-17,53}$ As well as the significant destabilization of $\mathrm{n} \pi^{*}$ transitions by hydrogen bond formation, we have identified N-H bending vibrations that further destabilise the $\mathrm{n} \pi^{*}$ states upon initial excitation, whilst conversely stabilising the $\pi \pi^{*}$ states.

The results of our dynamical simulations are strictly valid only in the gas phase, nonetheless some of the effects we have discussed are likely operative also for a GC pair in solution and within DNA. For example, we can expect that the $\mathrm{n} \pi^{*}$ states should also not be significantly populated within a duplex, since there is no reason to believe that a polar environment would increase their stability. Analogously, $\mathrm{G} \rightarrow \mathrm{C}(\mathrm{CT}) 1$ should also be significantly populated in solution. On the other hand, within a duplex, the population of additional CT states involving the adjacent, stacked, bases to $G$, can become competitive ${ }^{46,50,79}$ and we will investigate the interplay of intra- and inter-strand CT states in future work.

As shown in the present contribution, the FrD-LVC approach can thus provide many interesting insights into complex phenomena involving both intra- and inter-chromophoric excited states, in a fully quantum dynamical picture. Furthermore, in cases when an LVC PES does not suffice to completely describe the dynamics, it can give useful indications on the relative importance of the different excited states and be used as a 'first' check before applying more computationally expensive dynamical calculations, such as the 'on-the fly' methods, on reduced dimensionality systems. Alternatively, the FrD-LVC approach can be used as a 
building block onto which a more sophisticated model may be constructed, such as by considering anharmonic or dissociative coordinates like the proton transfer process in $\mathrm{GC}$, or inclusion of solvent effects, ${ }^{80,81}$ and we intend to explore both of these avenues in future work.

Acknowledgement This project has received funding from the European Union's Horizon 2020 Research and Innovation Programme under the Marie Sklodowska-Curie grant agreement No 765266 (LightDyNAmics).

\section{Supporting Information Avail- able}

Numerical details of the diabatization scheme in TD-DFT and calculation of absorption spectra. Electronic structure data including NTOs, normal mode displacement vectors and monomer vs dimer energies. Characterisation of the FrDLVC models including energies, couplings, and eigenvectors of adiabatic FrD-LVC states at the FC point and various excited state minima. Additional dynamics results including effect of deuteration, electronic only dynamics, dependence on $\mathrm{CT}-\pi \pi^{*}$ diabatic energy gap, and time-dependence of energies and couplings. Comparison of absorption spectra to experiment. Test calculation with larger basis set.

\section{References}

(1) Improta, R.; Santoro, F.; Blancafort, L. Quantum mechanical studies on the photophysics and the photochemistry of nucleic acids and nucleobases. Chem. Rev. 2016, 116, 3540 .

(2) Segatta, F.; Cupellini, L.; Garavelli, M.; Mennucci, B. Quantum Chemical Modeling of the Photoinduced Activity of Multichromophoric Biosystems. Chem. Rev. 2019, 119, 9361-9380.

(3) Curutchet, C.; Mennucci, B. Quantum Chemical Studies of Light Harvesting. Chem. Rev. 2017, 117, 294-343.
(4) Middleton, C. T.; de La Harpe, K.; Su, C.; Law, Y. K.; Crespo-Hernández, C. E.; Kohler, B. DNA Excited-State Dynamics: From Single Bases to the Double Helix. Annu. Rev. Phys. Chem. 2009, 60, 217239.

(5) Mirkovic, T.; Ostroumov, E. E.; Anna, J. M.; van Grondelle, R.; Govindjee,; Scholes, G. D. Light Absorption and Energy Transfer in the Antenna Complexes of Photosynthetic Organisms. Chem. Rev. 2017, 117, 249-293.

(6) Brixner, T.; Hildner, R.; Köhler, J.; Lambert, C.; Würthner, F. Exciton Transport in Molecular Aggregates - From Natural Antennas to Synthetic Chromophore Systems. Adv. Energy Mater. 2017, 7, 1700236 .

(7) Schröter, M.; Ivanov, S.; Schulze, J.; Polyutov, S.; Yan, Y.; Pullerits, T.; Kühn, O. Exciton-vibrational coupling in the dynamics and spectroscopy of Frenkel excitons in molecular aggregates. Phys. Rep. 2015, 567, 1 - 78 .

(8) Popp, W.; Brey, D.; Binder, R.; Burghardt, I. Quantum Dynamics of Exciton Transport and Dissociation in Multichromophoric Systems. Annu. Rev. Phys. Chem. 2021, 72, 591-616.

(9) Polkehn, M.; Eisenbrandt, P.; Tamura, H.; Burghardt, I. Quantum dynamical studies of ultrafast charge separation in nanostructured organic polymer materials: Effects of vibronic interactions and molecular packing. Int. J. Quantum Chem. 2018, 118, e25502.

(10) Hestand, N. J.; Spano, F. C. Expanded Theory of H- and J-Molecular Aggregates: The Effects of Vibronic Coupling and Intermolecular Charge Transfer. Chem. Rev. 2018, 118, 7069-7163.

(11) Cupellini, L.; Corbella, M.; Mennucci, B.; Curutchet, C. Electronic energy transfer in biomacromolecules. WIREs Comput. Mol. Sci. 2019, 9, e1392. 
(12) Köppel, H.; Domcke, W.; Cederbaum, L. S. Multimode Molecular Dynamics Beyond the Born-Oppenheimer Approximation. Adv. Chem. Phys. 1984, $57,59$.

(13) Liu, Y.; Cerezo, J.; Lin, N.; Zhao, X.; Improta, R.; Santoro, F. Comparison of the results of a mean-field mixed quantum/classical method with full quantum predictions for nonadiabatic dynamics: application to the $\pi \pi^{*} / n \pi^{*}$ decay of thymine. Theor. Chem. Acc. 2018, 137, 40 .

(14) Liu, Y.; Martínez Fernández, L.; Cerezo, J.; Prampolini, G.; Improta, R.; Santoro, F. Multistate coupled quantum dynamics of photoexcited cytosine in gasphase: Nonadiabatic absorption spectrum and ultrafast internal conversions. Chem. Phys. 2018, 515, 452-463.

(15) Yaghoubi Jouybari, M.; Liu, Y.; Improta, R.; Santoro, F. Ultrafast Dynamics of the Two Lowest Bright Excited States of Cytosine and 1-Methylcytosine: A Quantum Dynamical Study. J. Chem. Theory Comput. 2020, 16, 5792-5808.

(16) Yaghoubi Jouybari, M.; Liu, Y.; Improta, R.; Santoro, F. Quantum dynamics of the $\pi \pi^{*} / \mathrm{n} \pi^{*}$ decay of the epigenetic nucleobase 1,5-dimethyl-cytosine in the gas phase. Phys. Chem. Chem. Phys. 2020, 22, 26525-26535.

(17) Green, J. A.; Yaghoubi Jouybari, M.; Aranda, D.; Improta, R.; Santoro, F. Nonadiabatic Absorption Spectra and Ultrafast Dynamics of DNA and RNA Photoexcited Nucleobases. Molecules 2021, 26.

(18) Aleotti, F.; Aranda, D.; Yaghoubi Jouybari, M.; Garavelli, M.; Nenov, A.; Santoro, F. Parameterization of a linear vibronic coupling model with multiconfigurational electronic structure methods to study the quantum dynamics of photoexcited pyrene. J. Chem. Phys. 2021, 154, 104106.

(19) Aranda, D.; Santoro, F. Vibronic Spectra of $\pi$-Conjugated Systems with a Multitude of Coupled States. A Protocol Based on Linear Vibronic Coupling Models and Quantum Dynamics Tested on Hexahelicene. J. Chem. Theory Comput. 2021, 17, 1691-1700.

(20) Plasser, F.; Gómez, S.; Menger, M. F. S. J.; Mai, S.; González, L. Highly efficient surface hopping dynamics using a linear vibronic coupling model. Phys. Chem. Chem. Phys. 2019, 21, 57-69.

(21) Fumanal, M.; Gindensperger, E.; Daniel, C. Ultrafast Intersystem Crossing vs Internal Conversion in $\alpha$-Diimine Transition Metal Complexes: Quantum Evidence. J. Phys. Chem. Lett. 2018, 9, 5189-5195.

(22) Popp, W.; Polkehn, M.; Hughes, K. H.; Martinazzo, R.; Burghardt, I. Vibronic coupling models for donor-acceptor aggregates using an effective-mode scheme: Application to mixed Frenkel and chargetransfer excitons in oligothiophene aggregates. J. Chem. Phys. 2019, 150, 244114.

(23) Popp, W.; Polkehn, M.; Binder, R.; Burghardt, I. Coherent Charge Transfer Exciton Formation in Regioregular P3HT: A Quantum Dynamical Study. J. Phys. Chem. Lett. 2019, 10, 3326-3332.

(24) Polkehn, M.; Tamura, H.; Burghardt, I. Impact of charge-transfer excitons in regioregular polythiophene on the charge separation at polythiophene-fullerene heterojunctions. J. Phys. B: At., Mol. Opt. Phys. 2017, 51, 014003.

(25) Huix-Rotllant, M.; Tamura, H.; Burghardt, I. Concurrent Effects of Delocalization and Internal Conversion Tune Charge Separation at Regioregular Polythiophene-Fullerene Heterojunctions. J. Phys. Chem. Lett. 2015, 6, 1702-1708. 
(26) Tamura, H.; Burghardt, I. Ultrafast Charge Separation in Organic Photovoltaics Enhanced by Charge Delocalization and Vibronically Hot Exciton Dissociation. J. Am. Chem. Soc. 2013, 135, 16364-16367.

(27) Tamura, H.; Martinazzo, R.; Ruckenbauer, M.; Burghardt, I. Quantum dynamics of ultrafast charge transfer at an oligothiophene-fullerene heterojunction. J. Chem. Phys. 2012, 137, 22A540.

(28) Tamura, H.; Burghardt, I.; Tsukada, M. Exciton Dissociation at Thiophene/Fullerene Interfaces: The Electronic Structures and Quantum Dynamics. J. Phys. Chem. C 2011, 115, 10205-10210.

(29) Spencer, J.; Gajdos, F.; Blumberger, J. FOB-SH: Fragment orbital-based surface hopping for charge carrier transport in organic and biological molecules and materials. J. Chem. Phys. 2016, 145, 064102.

(30) Ghosh, S.; Giannini, S.; Lively, K.; Blumberger, J. Nonadiabatic dynamics with quantum nuclei: simulating charge transfer with ring polymer surface hopping. Faraday Discuss. 2020, 221, 501-525.

(31) Nebgen, B.; Prezhdo, O. V. Fragment Molecular Orbital Nonadiabatic Molecular Dynamics for Condensed Phase Systems. J. Phys. Chem. A 2016, 120, 72057212 .

(32) Sobolewski, A. L.; Domcke, W. Ab initio studies on the photophysics of the guanine-cytosine base pair. Phys. Chem. Chem. Phys. 2004, 6, 2763-2771.

(33) Sobolewski, A. L.; Domcke, W.; Hättig, C. Tautomeric selectivity of the excited-state lifetime of guanine/cytosine base pairs: The role of electron-driven proton-transfer processes. Proc. Natl. Acad. Sci. U.S.A 2005, 102, 17903-17906.
(34) Markwick, P. R. L.; Doltsinis, N. L. Ultrafast repair of irradiated DNA: Nonadiabatic ab initio simulations of the guanine-cytosine photocycle. J. Chem. Phys. 2007, 126, 175102.

(35) Markwick, P. R. L.; Doltsinis, N. L. Probing Irradiation Induced DNA Damage Mechanisms Using Excited State CarParrinello Molecular Dynamics. J. Chem. Phys. 2007, 126, 045104.

(36) Alexandrova, A. N.; Tully, J. C.; Granucci, G. Photochemistry of DNA Fragments via Semiclassical Nonadiabatic Dynamics. J. Phys. Chem. B 2010, 114, 12116-12128.

(37) Yamazaki, S.; Taketsugu, T. Photoreaction channels of the guanine-cytosine base pair explored by long-range corrected TDDFT calculations. Phys. Chem. Chem. Phys. 2012, 14, 8866-8877.

(38) Francés-Monerris, A.; Segarra-Martí, J.; Merchán, M.; Roca-Sanjuán, D. Theoretical study on the excited-state $\pi$ stacking versus intermolecular hydrogentransfer processes in the guaninecytosine/cytosine trimer. Theor. Chem. Acc. 2016, 135, 31.

(39) Groenhof, G.; Schäfer, L. V.; BoggioPasqua, M.; Goette, M.; Grubmüller, H.; Robb, M. A. Ultrafast Deactivation of an Excited Cytosine-Guanine Base Pair in DNA. J. Am. Chem. Soc. 2007, 129, 6812-6819.

(40) Sauri, V.; Gobbo, J. P.; SerranoPérez, J. J.; Lundberg, M.; Coto, P. B.; Serrano-Andrés, L.; Borin, A. C.; Lindh, R.; Merchán, M.; RocaSanjuán, D. Proton/Hydrogen Transfer Mechanisms in the Guanine-Cytosine Base Pair: Photostability and Tautomerism. J. Chem. Theory Comput. 2013, 9, 481-496.

(41) Szkaradek, K. E.; Stadlbauer, P.; Šponer, J.; Góra, R. W.; Szabla, R. 
UV-induced hydrogen transfer in DNA base pairs promoted by dark $\mathrm{n} \pi^{*}$ states. Chem. Commun. 2020, 56, 201-204.

(42) Schwalb, N. K.; Temps, F. Ultrafast Electronic Relaxation in Guanosine is Promoted by Hydrogen Bonding with Cytidine. J. Am. Chem. Soc. 2007, 129, 9272.

(43) Schwalb, N. K.; Michalak, T.; Temps, F. Ultrashort Fluorescence Lifetimes of Hydrogen-Bonded Base Pairs of Guanosine and Cytidine in Solution. J. Phys. Chem. B 2009, 113, 16365.

(44) Biemann, L.; Kovalenko, S. A.; Kleinermanns, K.; Mahrwald, R.; Markert, M.; Improta, R. Excited State Proton Transfer is Not Involved in the Ultrafast Deactivation of Guanine-Cytosine Pair in Solution. J. Am. Chem. Soc. 2011, 133, 19664.

(45) Röttger, K.; Marroux, H. J. B.; Grubb, M. P.; Coulter, P. M.; Bohnke, H.; Henderson, A. S.; Galan, M. C.; Temps, F.; Orr-Ewing, A. J.; Roberts, G. M. Ultraviolet Absorption Induces Hydrogen-Atom Transfer in G.C Watson-Crick DNA Base Pairs in Solution. Angew. Chem., Int. Ed. 2015, $54,14719$.

(46) Martinez-Fernandez, L.; Prampolini, G.; Cerezo, J.; Liu, Y.; Santoro, F.; Improta, R. Solvent effect on the energetics of proton coupled electron transfer in guanine-cytosine pair in chloroform by mixed explicit and implicit solvation models. Chem. Phys. 2018, 515, 493 - 501.

(47) Francés-Monerris, A.; Gattuso, H.; RocaSanjuán, D.; Tuñón, I. n.; Marazzi, M.; Dumont, E.; Monari, A. Dynamics of the excited-state hydrogen transfer in a $(\mathrm{dG}) \cdot(\mathrm{dC})$ homopolymer: intrinsic photostability of DNA. Chem. Sci. 2018, 9, 7902-7911.

(48) Martinez-Fernandez, L.; Improta, R. Photoactivated proton coupled electron transfer in DNA: insights from quantum me- chanical calculations. Faraday Discuss. 2018, 20\%, 199-216.

(49) Bucher, D. B.; Schlueter, A.; Carell, T.; Zinth, W. Watson-Crick Base Pairing Controls Excited-State Decay in Natural DNA. Angew. Chem., Int. Ed. 2014, 53, 11366 .

(50) Zhang, Y.; de La Harpe, K.; Beckstead, A. A.; Improta, R.; Kohler, B. UV-induced proton transfer between DNA strands. J. Am. Chem. Soc. 2015, 137, 7059 .

(51) Zhang, Y.; de La Harpe, K.; Beckstead, A. A.; Martinez-Fernandez, L.; Improta, R.; Kohler, B. Excited-state dynamics of DNA duplexes with different H-bonding motifs. J. Phys. Chem. Lett. 2016, \%, 950.

(52) Abo-Riziq, A.; Grace, L.; Nir, E.; Kabelac, M.; Hobza, P.; de Vries, M. S. Photochemical selectivity in guanine-cytosine base-pair structures. Proc. Natl. Acad. Sci. U.S.A 2005, 102, 20-23.

(53) Mai, S.; Marquetand, P.; Richter, M.; González-Vázquez, J.; González, L. Singlet and triplet excited-state dynamics study of the keto and enol tautomers of cytosine. ChemPhysChem 2013, 14, 29202931.

(54) Röttger, K.; Marroux, H. J. B.; Böhnke, H.; Morris, D. T. J.; Voice, A. T.; Temps, F.; Roberts, G. M.; OrrEwing, A. J. Probing the excited state relaxation dynamics of pyrimidine nucleosides in chloroform solution. Faraday Discuss. 2016, 194, 683-708.

(55) Quinn, S.; Doorley, G. W.; Watson, G. W.; Cowan, A. J.; George, M. W.; Parker, A. W.; Ronayne, K. L.; Towrie, M.; Kelly, J. M. Ultrafast IR spectroscopy of the short-lived transients formed by UV excitation of cytosine derivatives. Chem. Commun. 2007, 2130. 
(56) Wang, X.; Zhou, Z.; Tang, Y.; Chen, J.; Zhong, D.; Xu, J. Excited State Decay Pathways of 2'-Deoxy-5-methylcytidine and Deoxycytidine Revisited in Solution: A Comprehensive Kinetic Study by Femtosecond Transient Absorption. J. Phys. Chem. B 2018, 122, 7027-7037.

(57) Doorley, G. W.; McGovern, D. A.; George, M. W.; Towrie, M.; Parker, A. W.; Kelly, J. M.; Quinn, S. J. Picosecond Transient Infrared Study of the Ultrafast Deactivation Processes of Electronically Excited B-DNA and Z-DNA Forms of [poly $(\mathrm{dG}-\mathrm{dC})] 2$. Angew. Chem., Int. Ed. 2009, 48, 123-127.

(58) Mai, S.; Marquetand, P.; Richter, M.; González-Vazquez, J.; González, L. Singlet and Triplet Excited-State Dynamics Study of the Keto and Enol Tautomers of Cytosine. ChemPhysChem 2013, 14, 2920-2931.

(59) Richter, M.; Marquetand, P.; GonzálezVázquez, J.; Sola, I.; González, L. Femtosecond Intersystem Crossing in the DNA Nucleobase Cytosine. J. Phys. Chem. Lett. 2012, 3, 3090-3095.

(60) Mai, S.; Richter, M.; Marquetand, P.; González, L. Excitation of Nucleobases from a Computational Perspective II: Dynamics. Photoinduced Phenomena in $\mathrm{Nu}-$ cleic Acids I: Nucleobases in the Gas Phase and in Solvents. 2015; pp 99-153.

(61) Yanai, T.; Tew, D.; Handy, N. A new hybrid exchange-correlation functional using the Coulomb-attenuating method (CAM-B3LYP). Chem. Phys. Lett. 2004, 393, 51.

(62) Chai, J.-D.; Head-Gordon, M. Long-range corrected hybrid density functionals with damped atom-atom dispersion corrections. Phys. Chem. Chem. Phys. 2008, 10, 6615-6620.

(63) Green, J. A.; Asha, H.; Santoro, F.; Improta, R. Excitonic Model for Strongly
Coupled Multichromophoric Systems: The Electronic Circular Dichroism Spectra of Guanine Quadruplexes as Test Cases. J. Chem. Theory Comput. 2021, 17, 405-415.

(64) Green, J. A.; Improta, R. Vibrations of the guanine-cytosine pair in chloroform: an anharmonic computational study. Phys. Chem. Chem. Phys. 2020, 22, 5509-5522.

(65) Neugebauer, J.; Baerends, E. J.; Nooijen, M. Vibronic coupling and double excitations in linear response time-dependent density functional calculations: Dipoleallowed states of N2. J. Chem. Phys. 2004, 121, 6155-6166.

(66) Frisch, M. J.; Trucks, G. W.; Schlegel, H. B.; Scuseria, G. E.; Robb, M. A.; Cheeseman, J. R.; Scalmani, G.; Barone, V.; Petersson, G. A.; Nakatsuji, H.; Li, X.; Caricato, M.; Marenich, A. V.; Bloino, J.; Janesko, B. G.; Gomperts, R.; Mennucci, B.; Hratchian, H. P.; Ortiz, J. V.; Izmaylov, A. F.; Sonnenberg, J. L.; Williams-Young, D.; Ding, F.; Lipparini, F.; Egidi, F.; Goings, J.; Peng, B.; Petrone, A.; Henderson, T.; Ranasinghe, D.; Zakrzewski, V. G.; Gao, J.; Rega, N.; Zheng, G.; Liang, W.; Hada, M.; Ehara, M.; Toyota, K.; Fukuda, R.; Hasegawa, J.; Ishida, M.; Nakajima, T.; Honda, Y.; Kitao, O.; Nakai, H.; Vreven, T.; Throssell, K.; Montgomery, J. A., Jr.; Peralta, J. E.; Ogliaro, F.; Bearpark, M. J.; Heyd, J. J.; Brothers, E. N.; Kudin, K. N.; Staroverov, V. N.; Keith, T. A.; Kobayashi, R.; Normand, J.; Raghavachari, K.; Rendell, A. P.; Burant, J. C.; Iyengar, S. S.; Tomasi, J.; Cossi, M.; Millam, J. M.; Klene, M.; Adamo, C.; Cammi, R.; Ochterski, J. W.; Martin, R. L.; Morokuma, K.; Farkas, O.; Foresman, J. B.; Fox, D. J. Gaussian 16 Revision B.01. 2016; Gaussian Inc. Wallingford CT. 
(67) Wang, H.; Thoss, M. Multilayer formulation of the multiconfiguration timedependent Hartree theory. J. Chem. Phys. 2003, 119, 1289-1299.

(68) Vendrell, O.; Meyer, H.-D. Multilayer multiconfiguration time-dependent Hartree method: Implementation and applications to a Henon-Heiles Hamiltonian and to pyrazine. J. Chem. Phys. 2011, $134,044135$.

(69) Wang, H. Multilayer Multiconfiguration Time-Dependent Hartree Theory. J. Phys. Chem. A 2015, 119, 7951-7965.

(70) Worth, G. A.; Giri, K.; Richings, G. W.; Beck, M. H.; Jäckle, A.; Meyer, H.-D. The QUANTICS Package, Version 1.1, (2015), University of Birmingham, Birmingham, U.K.

(71) Raab, A.; Worth, G. A.; Meyer, H.D.; Cederbaum, L. S. Molecular dynamics of pyrazine after excitation to the S2 electronic state using a realistic 24-mode model Hamiltonian. J. Chem. Phys. 1999, 110, 936-946.

(72) Szalay, P. G.; Watson, T.; Perera, A.; Lotrich, V.; Bartlett, R. J. Benchmark Studies on the Building Blocks of DNA. 3. Watson-Crick and Stacked Base Pairs. J. Phys. Chem. A 2013, 117, 3149-3157.

(73) Improta, R.; Barone, V. Excited states behavior of nucleobases in solution: Insights from computational studies. Photoinduced Phenomena in Nucleic Acids I: Nucleobases in the Gas Phase and in Solvents. 2015; pp 329-358.

(74) Zhang, Y.; de La Harpe, K.; Kohl, F. R.; Kohler, B. Isotopic substitution affects excited state branching in a DNA duplex in aqueous solution. Chem. Commun. 2019, $55,4174-4177$.

(75) Martínez-Fernández, L.; Pepino, A. J.; Segarra-Martí, J.; Jovaišaité, J.; Vaya, I.;
Nenov, A.; Markovitsi, D.; Gustavsson, T.; Banyasz, A.; Garavelli, M.; Improta, R. Photophysics of Deoxycytidine and 5-Methyldeoxycytidine in Solution: A Comprehensive Picture by Quantum Mechanical Calculations and Femtosecond Fluorescence Spectroscopy. J. Am. Chem. Soc. 2017, 139, 7780-7791.

(76) Tully, J. C. Molecular Dynamics with Electronic Transitions. J. Chem. Phys. 1990, 93, 1061-1071.

(77) Lehr, A.; Gómez, S.; Parkes, M. A.; Worth, G. A. The role of vibronic coupling in the electronic spectroscopy of maleimide: a multi-mode and multi-state quantum dynamics study. Phys. Chem. Chem. Phys. 2020, 22, 25272-25283.

(78) Cerezo, J.; Aranda, D.; Avila Ferrer, F. J.; Prampolini, G.; Santoro, F. AdiabaticMolecular Dynamics Generalized Vertical Hessian Approach: A Mixed Quantum Classical Method To Compute Electronic Spectra of Flexible Molecules in the Condensed Phase. J. Chem. Theory Comput. 2020, 16, 1215-1231.

(79) Ko, C.; Hammes-Schiffer, S. ChargeTransfer Excited States and Proton Transfer in Model Guanine-Cytosine DNA Duplexes in Water. J. Phys. Chem. Lett. 2013, 4, 2540.

(80) Cerezo, J.; Liu, Y.; Lin, N.; Zhao, X.; Improta, R.; Santoro, F. Mixed Quantum/Classical Method for Nonadiabatic Quantum Dynamics in Explicit Solvent Models: The $\pi \pi^{*} / \mathrm{n} \pi^{*}$ Decay of Thymine in Water as a Test Case. J. Chem. Theory Comput. 2018, 14, 820-832.

(81) Santoro, F.; Green, J. A.; MartinezFernandez, L.; Cerezo, J.; Improta, R. Quantum and semiclassical dynamical studies of nonadiabatic processes in solution: achievements and perspectives. Phys. Chem. Chem. Phys. 2021, 23, 8181-8199. 